\title{
Stress Intensity Factor Solutions for Adhesive-Bonded Lap-Shear Specimens of Magnesium and Steel Sheets with and without Kinked Cracks for Fatigue Life Estimations
}

\author{
Wei-Jen Lai ${ }^{\mathrm{a}}$ and Jwo Pan ${ }^{\mathrm{b}}$ \\ ${ }^{a}$ Department of Materials Science and Engineering \\ The University of Michigan \\ Ann Arbor, MI, 48109, USA \\ ${ }^{\mathrm{b}}$ Department of Mechanical Engineering \\ The University of Michigan \\ Ann Arbor, MI, 48109, USA
}

August 24, 2014

\begin{abstract}
In this paper, stress intensity factor solutions for adhesive-bonded lap-shear specimens of magnesium alloy AZ31 and hot-dip-galvanized (HDG) mild steel sheets with and without kinked cracks are investigated for fatigue life estimations. First, the kinked fatigue crack failure mode of the adhesive-bonded lap-shear specimens is briefly reviewed. Then, the analytical global $J$ integral and effective stress intensity factor solutions for main cracks in lap-shear specimens of three dissimilar sheets under plane strain conditions are developed based on the beam bending theory. The global effective stress intensity factor solutions for the main cracks in the lap-shear specimens from the corresponding finite element analyses are then presented and validated by the analytical solutions. Next, the local stress intensity factor solutions for kinked cracks with the experimentally observed kink angle as functions of the kink length from the corresponding finite element analyses are presented and the computational solutions are also compared with the analytical solutions at small kink lengths. The results indicate that the computational local stress intensity factor solutions for kinked cracks approach to the analytical
\end{abstract}

Corresponding author. Tel.:+1-734-764-9404; fax:+1-734-647-3170.

Email addresses: weijen@umich.edu (W.-J. Lai), jwo@umich.edu (J. Pan). 
solutions as the kink length decreases to a small value and the kinked crack is under dominant mode I loading conditions. The computational results also indicate that the local stress intensity factor solutions at a small kink length of microstructural significance may be used as the stress intensity factor solutions for zero or near zero kink length for fatigue life estimations when the computational results are not available. The computational local stress intensity factor solutions are then adopted to estimate the fatigue lives of the lap-shear specimens based on a kinked crack growth model and available material constants for the Paris law. The fatigue life estimations are lower than the experimental results. However, the general trend of fatigue life estimations agrees with that of the experimental results.

\section{Keywords}

adhesive-bonded joint; dissimilar joint; lap-shear specimen; stress intensity factor solution; kinked crack; fatigue life estimation

\section{Nomenclature}

$\begin{array}{ll}\text { HDG } & \text { hot-dip-galvanized } \\ b & \text { specimen width } \\ L & \text { sheet length } \\ t_{u} & \text { thickness of sheet } u \\ t_{l 1} & \text { thickness of sheet } l 1 \\ t_{l 2} & \text { thickness of sheet } l 2 \\ V & \text { specimen overlap length } \\ w & \text { bonded width }\end{array}$




\begin{tabular}{|c|c|}
\hline E & Young's modulus \\
\hline$G$ & shear modulus \\
\hline$v$ & Poisson's ratio \\
\hline$\sigma$ & normal stress \\
\hline$J$ & $J$ integral \\
\hline$W$ & strain energy density function \\
\hline$W_{j}$ & strain energy density function for sheet $j$ \\
\hline$\Gamma$ & $J$ integration contour \\
\hline $\mathrm{d} s$ & differential arc length for the contour $\Gamma$ \\
\hline $\mathbf{n}$ & unit outward normal \\
\hline$n_{x}$ & $x$ component of the unit outward normal $\mathbf{n}$ \\
\hline $\mathbf{T}$ & traction vector \\
\hline$T_{i}\left(=\sigma_{i j} n_{j}\right)$ & components of the traction vector $\mathbf{T}$ \\
\hline $\mathbf{u}$ & displacement vector \\
\hline$u_{i}$ & components of the displacement vector $\mathbf{u}$ \\
\hline$\sigma_{i j}$ & stress components \\
\hline$\varepsilon_{i j}$ & strain components \\
\hline$E^{\prime}$ & Young's modulus under plane stress or plane strain conditions \\
\hline$\sigma^{*}$ & $\begin{array}{l}\text { normal structural stress to satisfy the equilibrium, and continuity of the } \\
\text { strain and strain gradient }\end{array}$ \\
\hline$\delta_{u}$ & ratio of the sheet $u$ thickness to the total sheet thickness \\
\hline$l 1$ & ratio of the sheet $l 1$ thickness to the total sheet thickness \\
\hline
\end{tabular}




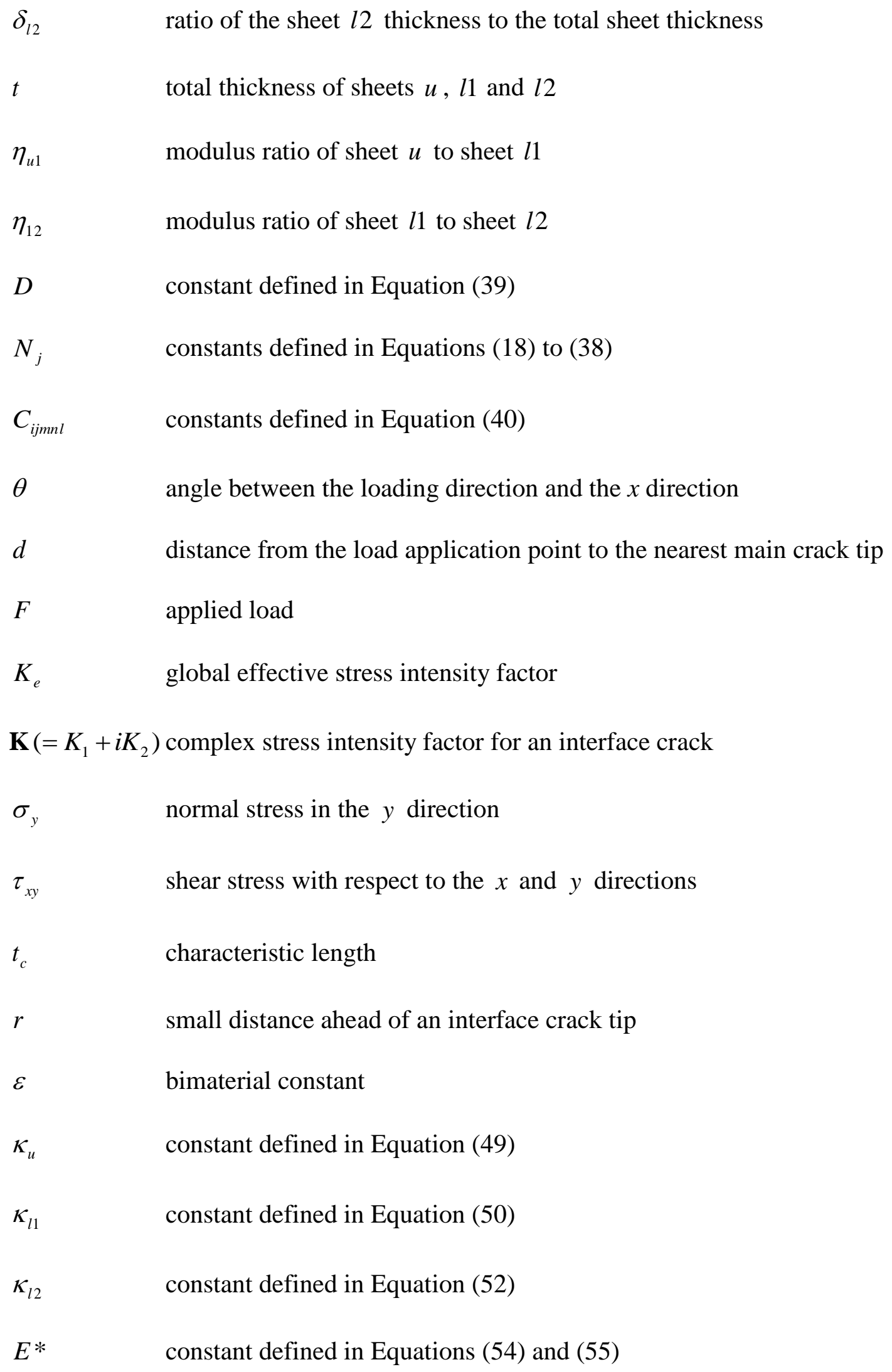


$K_{I}, K_{I I} \quad$ conventional mode I and II stress intensity factors

$\mathbf{K}^{A}\left(=K_{1}^{A}+i K_{2}^{A}\right) \quad$ complex stress intensity factor for an interface crack obtained from

\section{ABAQUS}

$F_{1}, F_{2}, F_{e} \quad$ dimensionless geometric functions for main cracks

a kink length

$\varphi \quad$ kink angle

$k_{I}, k_{I I} \quad$ local stress intensity factors

$k_{e} \quad$ local effective stress intensity factor

$\alpha, \beta \quad$ Dundurs' parameters

$c, d \quad$ complex functions of $\alpha, \beta$ and $\varphi$

$f_{I}, f_{I I}, f_{e} \quad$ dimensionless geometric functions for kinked cracks

$k_{e q} \quad$ equivalent stress intensity factor for the Paris law

$\gamma \quad$ material constant for the Paris law

$N \quad$ number of cycles

C material constant for the Paris law

$m \quad$ material constant for the Paris law

Subscripts and Superscripts

$u, l 1, l 2 \quad$ upper sheet, lower sheet 1 , and lower sheet 2

$i, o \quad$ inner and outer surfaces of the sheets with respect to the main crack 


\section{Introduction}

Lightweight materials such as advanced high strength steels, aluminum, and magnesium alloys have been replacing the traditional steel in the automotive industry to reduce the vehicle weight. Since magnesium alloys are much lighter than the steels commonly used in vehicles, using magnesium alloys could result in a substantial weight reduction. One of the major issues for introducing magnesium alloys into vehicle structures appears to be joining magnesium components to the existing steel structures. Joining magnesium alloys to steels is especially difficult due to the extreme difference in their melting temperatures and immiscibility of magnesium and iron (Santella et al. [1]). Melting magnesium alloys and steels together as might be done in resistance spot welding would vaporize magnesium and create unacceptable porosity in the weld nugget.

Solid state joining of magnesium alloys and steels offers a potential solution as the melting is either avoided or minimized. Ultrasonic welding is capable of joining similar and dissimilar materials. For joining similar materials using ultrasonic spot welding for automotive applications, researchers conducted research on processing conditions of joining similar aluminum sheets, for example, see Hetrick et al. [2], Jahn et al. [3] and Wright et al. [4]. For joining dissimilar sheets by ultrasonic spot welding, Watanabe et al. [5] conducted research on joining aluminum and steel sheets. Santella et al. [1] conducted research on joining magnesium to zinc-coated steel sheets by ultrasonic spot welding. The failure mode and fatigue behavior of the dissimilar ultrasonic spot welds in lap-shear specimens of magnesium AZ31B-H24 and hotdipped-galvanized mild steel sheets were investigated by Franklin et al. [6] and Lai et al. [7] under quasi-static and cyclic loading conditions. 
Lai et al. [7] explored the use of adhesive to increase the joint stiffness and to improve the fatigue performance of ultrasonic spot welds of the magnesium and steel sheets. Lai et al. [7] applied a layer of adhesive between the magnesium and steel sheets and ultrasonic spot welded the dissimilar sheets before the curing of the adhesive. The failure mode and fatigue behavior of the weld-bonded joints were then compared with those of the adhesive-bonded and ultrasonic spot welded joints. The results indicated that the adhesive-bonded and weld-bonded joints have much higher fatigue lives at a given load range than those of the ultrasonic spot weld joints under cyclic lap-shear loading conditions. However, the weld-bonded joints do not appear to provide additional fatigue lives for a given load range since the ultrasonic spot welds between the magnesium and steel sheets appear to be not strong enough to increase the fatigue lives under cyclic loading conditions. The adhesive-bonded and weld-bonded specimens of magnesium and steel sheets of Lai et al. [7] failed from the interface and subinterface fatigue crack growth under low-cycle loading conditions and failed from the kinked fatigue crack growth through the magnesium sheet under high-cycle loading conditions. The ultrasonic spot welds in lap-shear specimens of magnesium and steel sheets also failed from the kinked fatigue crack growth through the magnesium sheets.

Franklin [8] machined the lap-shear specimens of ultrasonic spot welds of the magnesium and steel sheets into a dog-bone shaped profile. The ultrasonic welds in these dog-bone shaped lap-shear specimens of magnesium and steel sheets also failed from the kinked fatigue crack growth through the magnesium sheets. For fatigue life estimations of these ultrasonic welded and adhesive-bonded specimens based on the kinked fatigue crack growth model using the Paris law, one needs to obtain the stress intensity factor solutions for kinked cracks at various kink lengths including zero or near zero kink length. For a kink crack growing out from a main crack 
between similar materials, the analytical solutions of Cotterell and Rice [9] can be used to calculate the stress intensity factors at zero or near zero kink length. However, analytical solutions for interface cracks between dissimilar materials of He and Hutchinson [10] are functions of the kink length, and the stress intensity factor solutions for kinked cracks growing out from a main crack between dissimilar materials at "zero" kink length cannot be determined.

In order to determine the fatigue life of the adhesive-bonded lap-shear specimen of dissimilar sheets based on a kinked fatigue crack growth model for kinked cracks growing out from an interface between dissimilar sheets, stress intensity factor solutions for kinked cracks with zero (or near zero) and finite kink lengths are needed. For small kink lengths, the analytical solution of He and Hutchinson [10] could be applicable. However, the range of the kink length, where the analytical stress intensity factor solutions are applicable, is not clear. For finite kink lengths, the stress intensity factor solutions can be obtained by finite element analyses. In this paper, the kinked fatigue crack failure mode of the adhesive-bonded lap-shear specimen of Lai et al. [7] is first reviewed briefly. Then, the analytical global $J$ integral and effective stress intensity factor solutions for main cracks in lap-shear specimens of three dissimilar sheets under plane strain conditions are developed based on the beam bending theory. The global effective stress intensity factor solutions for the main cracks in the lap-shear specimens from the corresponding finite element analyses are then presented and compared with the analytical solutions. Next, the local stress intensity factor solutions for kinked cracks with the experimentally observed kink angle as functions of the kink length from the corresponding finite element analyses are presented. The computational results are also compared with the analytical solutions for small kink lengths to determine the range of the kink length where the analytical solutions are applicable. The experimentally observed kink angle is then compared with the 
analytical solution. Based on the computational results, the local stress intensity factor solutions at a small kink length of microstructural significance, used as the stress intensity factor solutions at zero or near zero kink length, and the computational local stress intensity factor solutions at various finite kink lengths are adopted to estimate the fatigue lives of the lap-shear specimens based on a kinked crack growth model. The fatigue life estimations are then compared with the experimental results. Finally, some conclusions are made.

\section{Failure mode of adhesive-bonded lap-shear specimen of magnesium and steel sheets}

The experiment and the relevant results of adhesive-bonded lap-shear specimens of magnesium and steel sheets of Lai et al. [7] are briefly reviewed in this section. Magnesium AZ31B-H24 and hot-dip-galvanized (HDG) mild steel sheets with the thicknesses of $1.6 \mathrm{~mm}$ and $0.8 \mathrm{~mm}$, respectively, were used in Lai et

al. [7]. The lap-shear specimen was made using a $30 \mathrm{~mm} \times 100 \mathrm{~mm}$ magnesium sheet and a $30 \mathrm{~mm} \times 100 \mathrm{~mm}$ HDG steel sheet with a $30 \mathrm{~mm} \times 40 \mathrm{~mm}$ overlap area. The magnesium and steel sheets were bonded by BETAMATE ${ }^{\text {TM }} 73305$, a heat curing epoxy adhesive. Figures 1(a) and 1(b) show the top and bottom views of an adhesive-bonded lap-shear specimen of magnesium and steel sheets. Prior to applying the adhesive, two tapes were placed on the magnesium and steel sheets to form two preformed cracks between the sheets and the adhesive, as shown in

Figures 1(a) and 1(b). The locations of the tape tips are indicated in Figure 1(c). The tape tips are $5 \mathrm{~mm}$ from the edges of the overlap area. The adhesive thickness was controlled by placing a few 0.3-mm zirconia balls on 
the bonded surfaces such that the final adhesive thickness was $0.3 \mathrm{~mm}$. The specimen was then heated to the curing temperature to join the sheets. The area joined by the adhesive is $30 \mathrm{~mm} \times 30 \mathrm{~mm}$, which is smaller than the overlap area of the two sheets.

Figure 1(c) shows a schematic of the adhesive-bonded lap-shear specimen with the applied loads shown as the bold arrows. Two doublers were used to align the load. As schematically shown in Figure 1(c), the load is applied to the right on the lower surface of the upper right sheet and to the left on the upper surface of the lower left sheet. The specimen has a width $b$ and an overall length $L$ for the magnesium and steel sheets. The specimen has the thickness $t_{u}$ for the upper magnesium sheet, thickness $t_{l 1}$ for the adhesive layer, and thickness $t_{l 2}$ for the steel sheet. The specimen has an overlap length $V$. The bonded width $w$ is defined as the distance between two tape or crack tips as shown in Figure 1(c).

Figure 2(a) shows a micrograph of the cross section of a failed adhesive-bonded lap-shear specimen at the fatigue life of $3.6 \times 10^{4}$ cycles under the load range of $4,570 \mathrm{~N}$ with the load ratio $\mathrm{R}=0.1$. The applied load is shown by the two bold arrows. As shown in the figure, the fatigue crack was initiated at the right crack tip and grew into the magnesium sheet at an angle of about $30^{\circ}$ first and then grew vertically upward. Figure 2(b) shows a close-up view near the right crack tip. It is clearly seen that the tape tip, which is idealized as the crack tip, is where the fatigue crack started and grew into the magnesium sheet at an angle of about $30^{\circ}$. Hence, the fatigue crack can be treated as a kinked crack emanating from the right main crack tip. The kink angles observed in the failed adhesive-bonded lap-shear specimens of magnesium and steel sheets under 
cyclic loading conditions are all close to $30^{\circ}$. Detailed experimental procedures and fatigue test results can be found in Lai et al. [7].

\section{Analytical global $J$ integral solutions for main cracks}

The analytical $J$ integral solutions for main cracks between two dissimilar sheets was derived by Zhang [11] using the beam bending theory. However, one of the two preformed main cracks in the adhesive-bonded specimens is between the upper magnesium sheet and the middle adhesive layer. The other preformed main crack is between the lower steel sheet and the middle adhesive layer. Therefore, the analytical $J$ integral solutions for main cracks between two dissimilar sheets of Zhang [11] are not applicable. In this investigation, the $J$ integral solutions for a main crack in three dissimilar sheets are developed. Figure 3(a) shows a main crack between sheets $u$ and $l 1$ in three dissimilar sheets of $u, l 1$ and $l 2$. The three sheets are well bonded with each other and are assumed to be isotropic and linear elastic with Young's modulus $E_{u}$, shear modulus $G_{u}$ and Poisson's ratio $v_{u}$ for the upper sheet $u$ with the thickness $t_{u}, E_{l 1}$, $G_{l 1}$ and $v_{l 1}$ for the lower sheet $l 1$ with the thickness $t_{l 1}$, and $E_{l 2}, G_{l 2}$ and $v_{l 2}$ for the lower sheet $l 2$ with the thickness $t_{l 2}$. For the normal stresses $\sigma_{u i}, \sigma_{u o}, \sigma_{l l i}, \sigma_{l 1 o}, \sigma_{l 2 i}$ and $\sigma_{l 2 o}$ as shown in Figure 3(a), the subscripts $u, l 1$ and $l 2$ indicate the upper sheet, the lower sheet 1 and the lower sheet 2, respectively, and the subscripts $i$ and $o$ indicate the inner and outer surfaces of the sheets, respectively, with respect to the main crack. The coordinate system is also shown in the figure. The bonded width $w$ is assumed to be much larger than the thicknesses of the sheets so that the beam bending theory is applicable.

According to Rice [12], the $J$ integral solutions can be expressed as 


$$
J=\int_{\Gamma}\left(W n_{x}-T_{i} \frac{\partial u_{i}}{\partial x}\right) \mathrm{d} s, i=x, y
$$

where $\Gamma$ represents a contour counterclockwise from the lower crack face to the upper crack face, $\mathrm{d} s$ represents the differential arc length of the contour $\Gamma, n_{x}$ represents the $x$ component of the unit outward normal $\mathbf{n}$ to the differential arc length $\mathrm{d} s, T_{i}\left(=\sigma_{i j} n_{j}\right)$ represents the components of the traction vector $\mathbf{T}$ on the differential arc length $\mathrm{d} s$, and $u_{i}$ represents the components of the displacement vector $\mathbf{u}$. In Equation (1), the strain energy density $W$ is defined as

$$
W=\int_{0}^{\varepsilon_{i j}} \sigma_{i j} \mathrm{~d} \varepsilon_{i j}(i, j=x, y)
$$

where $\sigma_{i j}$ are the stress components and $\varepsilon_{i j}$ are the strain components. Consider a main crack on the right side as shown in Figure 3(a), the path-independent integration contour $\Gamma$ is selected as ABCDEFGHI in this case. The $J$ integral is 0 on lines BC and FG because $n_{x}=0$ and $T_{i}=0$. Recently, the three-dimensional finite element computational results of Wang et al. [13, 14] and Lin and Pan [15] for various types of spot weld specimens indicate that the structural stresses from the bending moments and the membrane forces provide the dominant contributions to the stress intensity factors along the nugget circumference whereas the structural stresses from the transverse shear forces may not have significant contributions. The $J$ integral on lines AB, $\mathrm{CD}, \mathrm{DE}, \mathrm{EF}, \mathrm{GH}$ and $\mathrm{HI}$ can therefore be written as

$$
J=-\left(\int_{A B} W_{u} \mathrm{~d} y+\int_{C D} W_{u} \mathrm{~d} y+\int_{D E} W_{l 1} \mathrm{~d} y+\int_{E F} W_{l 1} \mathrm{~d} y+\int_{G H} W_{l 2} \mathrm{~d} y+\int_{H I} W_{l 2} \mathrm{~d} y\right)
$$

with

$$
W_{j}=\frac{1}{2 E_{j}^{\prime}} \sigma_{x}^{2}(j=u, l 1, l 2)
$$


where $E_{j}^{\prime}=E_{j}$ under plane stress conditions and $E_{j}^{\prime}=E_{j} /\left(1-v_{j}^{2}\right)$ under plane strain

conditions. The stresses $\sigma_{x}$ on lines $\mathrm{AB}, \mathrm{GH}$ and $\mathrm{HI}$ can be determined directly from the applied load using the beam bending theory. The stresses $\sigma_{x}$ on lines $\mathrm{CD}, \mathrm{DE}$ and $\mathrm{EF}$ are determined by the six stresses $\sigma_{u i}^{*}, \sigma_{u o}^{*}, \sigma_{l 1 i}^{*}, \sigma_{l 1 o}^{*}, \sigma_{l 2 i}^{*}$ and $\sigma_{l 2 o}^{*}$ which can be determined from the linear strain distribution in the three dissimilar sheets according to the beam bending theory. To solve for the six stresses, six equations are derived based on the beam bending theory with the thickness ratios defined as

$\delta_{u}=t_{u} / t$

$\delta_{l 1}=t_{l 1} / t$

$\delta_{l 2}=t_{l 2} / t$

where

$t=t_{u}+t_{l 1}+t_{l 2}$

The Young's modulus ratios are defined as

$\eta_{u 1}=E_{u}^{\prime} / E^{\prime}{ }_{l 1}$

$\eta_{12}=E^{\prime}{ }_{l 1} / E^{\prime}{ }_{l 2}$

The six equations based on the beam bending theory are

$$
\begin{aligned}
& \delta_{l 2}\left(\sigma_{l 2 o}^{*}+\sigma_{l 2 i}^{*}\right)+\delta_{l 1}\left(\sigma_{l 1 o}^{*}+\sigma_{l 1 i}^{*}\right)+\delta_{u}\left(\sigma_{u i}^{*}+\sigma_{u o}^{*}\right) \\
& =\delta_{l 2}\left(\sigma_{l 2 o}+\sigma_{l 2 i}\right)+\delta_{l 1}\left(\sigma_{l 1 o}+\sigma_{l 1 i}\right)+\delta_{u}\left(\sigma_{u i}+\sigma_{u o}\right) \\
& \frac{1}{6} \delta_{l 2}\left[3 \delta_{l 1}\left(\sigma_{l 2 o}^{*}+\sigma_{l 2 i}^{*}\right)+\delta_{l 2}\left(2 \sigma_{l 2 o}^{*}+\sigma_{l 2 i}^{*}\right)\right]+\frac{1}{6} \delta_{l 1}^{2} \sigma_{l 1 i}^{*}+\frac{1}{3} \delta_{l 1}^{2} \sigma_{l 1 o}^{*}-\left(\frac{1}{3} \delta_{u}^{2} \sigma_{u o}^{*}+\frac{1}{6} \delta_{u}^{2} \sigma_{u i}^{*}\right) \\
& =\frac{1}{6} \delta_{l 2}\left[3 \delta_{l 1}\left(\sigma_{l 2 o}+\sigma_{l 2 i}\right)+\delta_{l 2}\left(2 \sigma_{l 2 o}+\sigma_{l 2 i}\right)\right]+\frac{1}{6} \delta_{l 1}^{2} \sigma_{l 1 i}+\frac{1}{3} \delta_{l 1}^{2} \sigma_{l 1 o}-\left(\frac{1}{3} \delta_{u}^{2} \sigma_{u o}+\frac{1}{6} \delta_{u}^{2} \sigma_{u i}\right)
\end{aligned}
$$




$$
\begin{aligned}
& \frac{\sigma_{u i}^{*}-\sigma_{u o}^{*}}{\delta_{u}}=\eta_{u 1} \frac{\sigma_{l 1 i}^{*}-\sigma_{l 1 o}^{*}}{\delta_{l 1}} \\
& \frac{\sigma_{l 1 i}^{*}-\sigma_{l 1 o}^{*}}{\delta_{l 1}}=\eta_{12} \frac{\sigma_{l 2 i}^{*}-\sigma_{l 2 o}^{*}}{\delta_{l 2}} \\
& \sigma_{u i}^{*}=\eta_{u 1} \sigma_{l 1 i}^{*} \\
& \sigma_{l 1 o}^{*}=\eta_{12} \sigma_{l 2 i}^{*}
\end{aligned}
$$

Equations (11) and (12) are from the force and moment equilibrium conditions, respectively. Equations (13) and (14) are from the linear strain distribution in the three dissimilar sheets. Equations (15) and (16) are from the strain continuity conditions at the two interfaces. Solving Equations (11) to (16) gives the six normal structural stresses $\sigma_{u i}^{*}, \sigma_{u o}^{*}, \sigma_{l 1 i}^{*}$, $\sigma_{l 1 o}^{*}, \sigma_{l 2 i}^{*}$ and $\sigma_{l 2 o}^{*}$. Substituting the six normal structural stresses $\sigma_{u i}^{*}, \sigma_{u o}^{*}, \sigma_{l 1 i}^{*}, \sigma_{l 1 o}^{*}, \sigma_{l 2 i}^{*}$ and $\sigma_{l 2 o}^{*}$ into Equation (4) and integrating over the contour $\Gamma$, the $J$ integral solutions for the main crack in three dissimilar sheets can be expressed as

$$
J=\frac{t}{6 E_{u}^{\prime} D}\left(\begin{array}{l}
N_{1} \sigma_{u i}^{2}+N_{2} \sigma_{u o}^{2}+N_{3} \sigma_{l 1 i}^{2}+N_{4} \sigma_{l 1 o}^{2}+N_{5} \sigma_{l 2 i}^{2}+N_{6} \sigma_{l 2 o}^{2} \\
+N_{7} \sigma_{u i} \sigma_{u o}+N_{8} \sigma_{u i} \sigma_{l 1 i}+N_{9} \sigma_{u i} \sigma_{l 1 o}+N_{10} \sigma_{u i} \sigma_{l 2 i}+N_{11} \sigma_{u i} \sigma_{l 2 o} \\
+N_{12} \sigma_{u o} \sigma_{l 1 i}+N_{13} \sigma_{u o} \sigma_{l 1 o}+N_{14} \sigma_{u o} \sigma_{l 2 i}+N_{15} \sigma_{u o} \sigma_{l 2 o} \\
+N_{16} \sigma_{l 1 i} \sigma_{l 1 o}+N_{17} \sigma_{l 1 i} \sigma_{l 2 i}+N_{18} \sigma_{l 1 i} \sigma_{l 2 o} \\
+N_{19} \sigma_{l 1 o} \sigma_{l 2 i}+N_{20} \sigma_{l 1 o} \sigma_{l 2 o} \\
+N_{21} \sigma_{l 2 i} \sigma_{l 2 o}
\end{array}\right)
$$

where

$$
\begin{aligned}
& N_{1}=C_{04100}+4 C_{31110}+6 C_{22110}+4 C_{13110}+C_{40120}+3 C_{21211}+3 C_{12211}+C_{03211}+6 C_{11311} \\
& +3 C_{02311}+3 C_{01411}+C_{30221}+3 C_{20321}+3 C_{10421} \\
& N_{2}=C_{04100}+4 C_{31110}+6 C_{22110}+4 C_{13110}+C_{40120}+3 C_{21211}+3 C_{12211}+C_{03211}+C_{30221} \\
& N_{3}=C_{14012}+C_{23011}+12 C_{31112}+12 C_{22112}+4 C_{13112}+12 C_{21212}+6 C_{12212}+4 C_{11312}+3 C_{40122} \\
& +3 C_{30222}+C_{20322}+C_{10423}
\end{aligned}
$$




$$
\begin{aligned}
& N_{4}=C_{14001}+3 C_{41011}+3 C_{32011}+C_{23011}+12 C_{31112}+12 C_{22112}+4 C_{13112}+12 C_{21212}+6 C_{12212} \\
& +4 C_{11312}+C_{20322}+C_{10423} \\
& N_{5}=C_{32021}+3 C_{23021}+3 C_{14021}+C_{41031}+3 C_{22122}+6 C_{13122}+3 C_{04122}+3 C_{12222}+3 C_{03222} \\
& +C_{02322}+4 C_{31132}+6 C_{21232}+4 C_{11332}+C_{01433} \\
& N_{6}=C_{32021}+C_{41031}+3 C_{22122}+3 C_{12222}+C_{02322}+4 C_{31132}+6 C_{21232}+4 C_{11332}+C_{01433} \\
& N_{7}=C_{04100}+4 C_{31110}+6 C_{22110}+4 C_{13110}+C_{40120}-6 C_{21211}-6 C_{12211}-2 C_{03211}-6 C_{11311} \\
& -3 C_{02311}-2 C_{30221}-3 C_{20321} \\
& N_{8}=-12 C_{31111}-15 C_{22111}-6 C_{13111}-4 C_{21211}-3 C_{12211}-3 C_{40121}-C_{30221}-C_{20322}-3 C_{10422} \\
& N_{9}=-6 C_{31111}-12 C_{22111}-6 C_{13111}-2 C_{21211}-3 C_{12211}+C_{30221}-2 C_{20322}-3 C_{10422} \\
& N_{10}=-3 C_{13111}-3 C_{04111}-C_{03211}+3 C_{31121}+3 C_{22121}+3 C_{21221}+2 C_{12221}-3 C_{11322}-C_{02322} \\
& -3 C_{01422}
\end{aligned}
$$$$
N_{11}=3 C_{13111}+C_{03211}+3 C_{31121}+6 C_{22121}+3 C_{21221}+4 C_{12221}-3 C_{11322}-2 C_{02322}+3 C_{01422}
$$$$
N_{12}=-12 C_{31111}-15 C_{22111}-6 C_{13111}-8 C_{21211}-6 C_{12211}-3 C_{40121}-2 C_{30221}+C_{20322}
$$$$
N_{13}=-6 C_{3111}-12 C_{22111}-6 C_{13111}-4 C_{21211}-6 C_{12211}+2 C_{30221}+2 C_{20322}
$$$$
N_{14}=-3 C_{13111}-3 C_{04111}-2 C_{03211}+3 C_{31121}+3 C_{22121}+6 C_{21221}+4 C_{12221}+3 C_{11322}+C_{02322}
$$$$
N_{15}=3 C_{13111}+2 C_{03211}+3 C_{31121}+6 C_{22121}+6 C_{21221}+8 C_{12221}+3 C_{11322}+2 C_{02322}
$$$$
N_{16}=C_{14001}-3 C_{32011}-2 C_{23011}+12 C_{31112}+12 C_{22112}+4 C_{13112}+12 C_{21212}+6 C_{12212}+4 C_{11312}
$$$$
-3 C_{30222}-2 C_{20322}+C_{10423}
$$$$
N_{17}=-2 C_{23011}-3 C_{14011}+C_{32021}-6 C_{31122}-2 C_{22122}-12 C_{21222}-3 C_{12222}-6 C_{11322}
$$$$
N_{18}=2 C_{23011}+2 C_{32021}-6 C_{31122}-4 C_{22122}-12 C_{21222}-6 C_{12222}-6 C_{11322}
$$$$
N_{19}=-C_{23011}-3 C_{14011}-3 C_{41021}-C_{32021}-12 C_{31122}-4 C_{22122}-15 C_{21222}-3 C_{12222}-6 C_{11322}
$$$$
N_{20}=C_{23011}-3 C_{41021}-2 C_{32021}-12 C_{31122}-8 C_{22122}-15 C_{21222}-6 C_{12222}-6 C_{11322}
$$ 
$N_{21}=-2 C_{32021}-3 C_{23021}+C_{41031}-6 C_{22122}-6 C_{13122}-6 C_{12222}-3 C_{03222}-2 C_{02322}+4 C_{31132}$

$+6 C_{21232}+4 C_{11332}+C_{01433}$

$D=C_{04000}+4 C_{31010}+6 C_{22010}+4 C_{13010}+C_{40020}+12 C_{2111}+12 C_{12111}+4 C_{03111}+12 C_{11211}$

$+6 C_{02211}+4 C_{01311}+4 C_{30121}+6 C_{20221}+4 C_{10321}+C_{00422}$

with

$C_{i j k l m}=\delta_{l 1}^{i} \delta_{l 2}^{j} \delta_{u}^{k} \eta_{12}^{l} \eta_{u 1}^{m}$

Figure 3(b) shows a schematic of the lap-shear specimen near the crack tips. Figure 4(a) shows a schematic of the lap-shear specimen with the applied load $F / b$. The positions of the two main crack tips are also shown in Figures 3(b) and 4(a). For the loading conditions shown in Figures 3(b) and 4(a), the normal structural stresses $\sigma_{u i}, \sigma_{u o}, \sigma_{l 1 i}, \sigma_{l 1 o}, \sigma_{l 2 i}$ and $\sigma_{l 2 o}$ for the right crack tip are

$$
\begin{aligned}
& \sigma_{u i, \text { right }}=\frac{4 F \cos \theta}{b t_{u}}+\frac{6 F d \sin \theta}{b t_{u}^{2}} \\
& \sigma_{u o, \text { right }}=-\frac{2 F \cos \theta}{b t_{u}}-\frac{6 F d \sin \theta}{b t_{u}^{2}} \\
& \sigma_{l 1 i, \text { right }}=0 \\
& \sigma_{l 1 o, \text { right }}=0 \\
& \sigma_{l 2 i, \text { right }}=0 \\
& \sigma_{l 2 o, \text { right }}=0
\end{aligned}
$$

where $\theta$ is the angle between the loading direction and the $x$ direction, $d$ is the distance from the load application point to the nearest main crack tip, and $F / b$ is the applied load per unit width as shown in Figure 4(a). The first and second terms in Equations (41) and (42) are due to the decomposed forces, $F \cos \theta$ and $F \sin \theta$, in the horizontal and vertical directions, 
respectively. Substituting Equations (41) to (46) into Equation (17) gives the value of the $J$ integral for the right crack tip.

The asymptotic in-plane stress field around a main crack tip is an oscillatory field that can be characterized by a complex stress intensity factor $\mathbf{K}\left(=K_{1}+i K_{2}, i=\sqrt{-1}\right)$ (Rice and Sih [16] and Rice [17]). The stresses $\sigma_{y}$ and $\tau_{x y}$ at a small distance $r$ ahead of a main crack tip are characterized by $\mathbf{K}$ as

$$
\sigma_{y}+i \tau_{x y}=\frac{K_{1}+i K_{2}}{\sqrt{2 \pi r}}\left(\frac{r}{t_{c}}\right)^{i \varepsilon}
$$

For the right crack as shown in Figure 3(b), the bimaterial constant $\varepsilon$ is defined as

$$
\varepsilon=\frac{1}{2 \pi} \ln \frac{\kappa_{u} / G_{u}+1 / G_{l 1}}{\kappa_{l 1} / G_{l 1}+1 / G_{u}}
$$

where

$$
\kappa_{u}=3-4 v_{u}
$$

and

$$
\kappa_{l 1}=3-4 v_{l 1}
$$

under plane strain conditions. For the left crack as shown in Figure 3(b), the bimaterial constant $\varepsilon$ is defined as

$$
\varepsilon=\frac{1}{2 \pi} \ln \frac{\kappa_{l 2} / G_{l 2}+1 / G_{l 1}}{\kappa_{l 1} / G_{l 1}+1 / G_{l 2}}
$$

where

$$
\kappa_{l 2}=3-4 v_{l 2}
$$

under plane strain conditions. In Equation $(47), t_{c}$ represents a characteristic length $[11,17]$. It should be noted that when the two materials are identical, $\varepsilon=0$. In this case, $K_{1}$ and $K_{2}$ in 
Equation (47) for the main crack become the conventional stress intensity factors $K_{I}$ and $K_{I I}$, respectively.

The $J$ integral solutions is directly related to the effective stress intensity factor $K_{e}$ for both the right and left cracks defined as

$$
K_{e}=\sqrt{K_{1}^{2}+K_{2}^{2}}=\sqrt{J \cosh ^{2}(\pi \varepsilon) E^{*}}
$$

where

$$
\frac{1}{E^{*}}=\frac{1}{2}\left(\frac{1}{E_{u}^{\prime}}+\frac{1}{E_{l 1}^{\prime}}\right)
$$

for the right crack and

$$
\frac{1}{E^{*}}=\frac{1}{2}\left(\frac{1}{E_{l 1}^{\prime}}+\frac{1}{E_{l 2}^{\prime}}\right)
$$

for the left crack.

\section{Computational global stress intensity factor solutions for main cracks}

Figure 4(a) shows a schematic of a two-dimensional finite element model of a lap-shear specimen with the boundary and loading conditions. The specimen has the upper sheet thickness $t_{u}$, the lower sheet 1 thickness $t_{l 1}$, and the lower sheet 2 thickness $t_{l 2}$, the length $L$, the overlap length $V$, and the bonded width $w$. The $x$-y coordinate system is shown in the figure. The left edge is fixed at the middle surface while the right edge has a concentrated force per unit width, $F / b$, applied at the middle surface.

The two-dimensional plane strain finite element model has $t_{u}=1.6 \mathrm{~mm}, t_{l 1}=0.3 \mathrm{~mm}$, $t_{l 2}=0.8 \mathrm{~mm}, L=100 \mathrm{~mm}$ and $V=40 \mathrm{~mm}$. The specimen width $b$ is $30 \mathrm{~mm}$, the angle $\theta$ between the loading direction and the $x$ direction is $0.107^{\circ}$, and the distance $d$ from the load 
application point to the nearest main crack tip is $65 \mathrm{~mm}$ based on the specimen geometry and the test setup in Lai et al. [7]. Note that the ratio of the bonded width $w$ to the upper sheet thickness $t_{u}$ is 37.5 for the specimens used in the experiments. The two-dimensional plane strain finite element model is used to obtain the global stress intensity factor solutions for calculating the local stress intensity factor solutions for kinked cracks. Figures 4(b) and 4(c) show the closed-up views of the meshes near the right and left main crack tips, respectively. All the materials are assumed to be isotropic and linear elastic. The top AZ31-H24 sheet is modeled with the Young's modulus $E_{u}=45 \mathrm{GPa}$ and the Poisson's ratio $v_{u}=0.35$. The middle adhesive layer is modeled with the Young's modulus $E_{l 1}=4.1 \mathrm{GPa}$ and the Poisson's ratio $v_{l 1}=0.36$. The bottom steel sheet is modeled with the Young's modulus $E_{l 2}=207 \mathrm{GPa}$ and the Poisson's ratio $v_{l 2}=0.3$. Second-order, isoparametric, quadrilateral, reduced integration, plane strain elements (CPE8R) were used in the model. The crack-tip elements were modified with collapsed nodes at the crack tip and the midside nodes on the sides were moved to the quarter points from the crack tip to model the $1 / \sqrt{r}$ singularity near the crack tip. The commercial finite element program ABAQUS [18] was employed to perform the computation.

It should be noted that the $K_{1}^{A}$ and $K_{2}^{A}$ of the complex stress intensity factor $\mathbf{K}^{A}$ $\left(=K_{1}^{A}+i K_{2}^{A}\right)$ obtained directly from ABAQUS [18] are defined such that the stresses $\sigma_{y}$ and $\tau_{x y}$ at a small distance $r$ ahead of a main crack tip are characterized by $\mathbf{K}^{A}$ as

$\sigma_{y}+i \tau_{x y}=\frac{K_{1}^{A}+i K_{2}^{A}}{\sqrt{2 \pi r}} r^{i \varepsilon}$

By comparing Equations (47) and (56), the $K_{1}$ and $K_{2}$ solutions can be related to the $K_{1}^{A}$ and $K_{2}^{A}$ solutions as 


$$
\begin{aligned}
& K_{1}=K_{1}^{A} \cos \left(\varepsilon \ln t_{c}\right)-K_{2}^{A} \sin \left(\varepsilon \ln t_{c}\right) \\
& K_{2}=K_{1}^{A} \sin \left(\varepsilon \ln t_{c}\right)+K_{2}^{A} \cos \left(\varepsilon \ln t_{c}\right)
\end{aligned}
$$

Here, the characteristic length $t_{c}$ is taken as the upper magnesium sheet thickness $t_{u}(=1.6 \mathrm{~mm})$ for calculating the $K_{1}$ and $K_{2}$ solutions. It is noted that the global effective stress intensity factor $K_{e}\left(=\sqrt{K_{1}^{2}+K_{2}^{2}}=\sqrt{\left(K_{1}^{A}\right)^{2}+\left(K_{2}^{A}\right)^{2}}\right)$ is independent of the selection of a characteristic length $t_{c}$. In this investigation, the computational $K_{1}$ and $K_{2}$ solutions for the interface cracks are obtained from Equations (57) and (58) with the $K_{1}^{A}$ and $K_{2}^{A}$ solutions obtained from ABAQUS [18].

The computational results can be expressed in the normalized forms as

$$
\begin{aligned}
& K_{1}=F_{1}\left(\delta_{u}, \delta_{l 1}, \delta_{l 2}, \eta_{u 1}, \eta_{12}\right) \cdot \frac{F}{b \sqrt{t_{u}}} \\
& K_{2}=F_{2}\left(\delta_{u}, \delta_{l 1}, \delta_{l 2}, \eta_{u 1}, \eta_{12}\right) \cdot \frac{F}{b \sqrt{t_{u}}} \\
& K_{e}=F_{e}\left(\delta_{u}, \delta_{l 1}, \delta_{l 2}, \eta_{u 1}, \eta_{12}\right) \cdot \frac{F}{b \sqrt{t_{u}}}
\end{aligned}
$$

where $F_{1}, F_{2}$ and $F_{e}$ are dimensionless geometric functions which depend on the thickness ratios and modulus ratios of the three sheets. It should be noted that the dimensionless geometric functions can be considered as the normalized stress intensity factor solutions. The values of the dimensionless geometric functions $F_{1}, F_{2}$ and $F_{e}$ and the ratio $F_{2} / F_{1}$ for the left and right main cracks are listed in Table 1. The values of the dimensionless geometric function $F_{e}$ obtained from the analytical solutions in Equations (17) and (53) for the left and right main cracks are also listed in the table. As listed in the table, the values of the dimensionless 
geometric function $F_{e}$ obtained from the finite element analyses and the analytical solutions for the left main crack are 0.625 and 0.622 , respectively. The values of the dimensionless geometric function $F_{e}$ obtained from the finite element analyses and the analytical solutions for the right main crack are 0.830 and 0.828 , respectively. The results show that the computational results agree very well with the analytical solutions with less than a small difference of $0.5 \%$.

\section{Analytical local stress intensity factor solutions for kinked cracks}

Figure 5 shows a schematic of a main crack and a kinked crack with the kink length $a$ and the kink angle $\varphi$. The schematic of the kinked crack is consistent with the kinked crack shown in Figure 2(a) by rotating $180^{\circ}$ either clockwise or counterclockwise. Here, $k_{I}$ and $k_{I I}$ represent the local stress intensity factor solutions for the kinked crack. The arrows shown in the figure represent the positive values of the local stress intensity factors $k_{I}$ and $k_{I I}$. The selection of the $x-y$ coordinate system with respect to the main crack tip is consistent with that in $\mathrm{He}$ and Hutchinson [10].

For kinked cracks that grow out from a main interface crack between dissimilar sheets, the $k_{I}$ and $k_{I I}$ solutions can be expressed as functions of the kink angle $\varphi$, the Dundurs' parameters $\alpha$ and $\beta$, and the global $K_{1}^{A}$ and $K_{2}^{A}$ solutions for the main crack when the kink length approaches to 0 . The local stress intensity factors $k_{I}$ and $k_{I I}$ are expressed in the complex form (He and Hutchinson [10]) based on the complex stress intensity factor $\mathbf{K}^{A}$ defined in Equation (56) as

$k_{I}+i k_{I I}=\left(c_{R}+i c_{I}\right)\left(K_{1}^{A}+i K_{2}^{A}\right) a^{i \varepsilon}+\left(d_{R}-i d_{I}\right)\left(K_{1}^{A}-i K_{2}^{A}\right) a^{-i \varepsilon}$ 
where $c_{R}, c_{I}, d_{R}$ and $d_{I}$ are the real and imaginary parts of the complex function $c$ and $d$. Both $c$ and $d$ are complex functions of $\alpha, \beta$ and $\varphi$. The Dundur's parameters $\alpha$ and $\beta$ are defined as

$$
\begin{gathered}
\alpha=\frac{\left(\kappa_{l}+1\right) G_{u}-\left(\kappa_{u}+1\right) G_{l}}{\left(\kappa_{l}+1\right) G_{u}+\left(\kappa_{u}+1\right) G_{l}} \\
\beta=\frac{\left(\kappa_{l}-1\right) G_{u}-\left(\kappa_{u}-1\right) G_{l}}{\left(\kappa_{l}+1\right) G_{u}+\left(\kappa_{u}+1\right) G_{l}}
\end{gathered}
$$

where the subscripts $u$ and $l$ represent the upper and lower sheets, respectively, following the notation shown in Figure 5. Equation (62) indicates that $k_{\mathrm{I}}$ and $k_{\mathrm{II}}$ depend on the kink length $a$ and the bimaterial constant $\varepsilon$. Note that Equation (62) is only applicable when the kink length approaches to 0. Based on Equation (62), $k_{\mathrm{I}}$ and $k_{\mathrm{II}}$ can be expressed as

$$
\begin{aligned}
& k_{I}=\left[-c_{I} \sin (\varepsilon \ln a)+c_{R} \cos (\varepsilon \ln a)-d_{I} \sin (\varepsilon \ln a)+d_{R} \cos (\varepsilon \ln a)\right] K_{1}^{A} \\
& +\left[-c_{I} \cos (\varepsilon \ln a)-c_{R} \sin (\varepsilon \ln a)-d_{I} \cos (\varepsilon \ln a)-d_{R} \sin (\varepsilon \ln a)\right] K_{2}^{A} \\
& k_{I I}=\left[c_{I} \cos (\varepsilon \ln a)+c_{R} \sin (\varepsilon \ln a)-d_{I} \cos (\varepsilon \ln a)-d_{R} \sin (\varepsilon \ln a)\right] K_{1}^{A} \\
& +\left[-c_{I} \sin (\varepsilon \ln a)+c_{R} \cos (\varepsilon \ln a)+d_{I} \sin (\varepsilon \ln a)-d_{R} \cos (\varepsilon \ln a)\right] K_{2}^{A}
\end{aligned}
$$

The global stress intensity factors $K_{1}^{A}$ and $K_{2}^{A}$ were obtained computationally as mentioned in the previous section. The functions $c_{R}, c_{I}, d_{R}$ and $d_{I}$ are tabulated in He and Hutchinson [19]. The values for $c$ and $d$ are extrapolated from that report. Based on the material properties used in the finite element analyses in section $4, c_{R}=1.026, c_{I}=-1.016, d_{R}=0.467$ and $d_{I}=-0.352$ for $\alpha=-0.832, \beta=-0.181$ and $\varepsilon=0.058$ for the right crack. As indicated in Equations (65) and (66), the values for the local stress intensity factor solutions depend on the kink length $a$ when the kink length approaches to 0 for $\varepsilon \neq 0$. The dependence of the solutions 
on the kink length $a$ presents a challenge to evaluate the local stress intensity factors $k_{\mathrm{I}}$ and $k_{\mathrm{II}}$ at zero or near zero kink length for estimation of fatigue lives based on a fatigue crack growth model, even though the oscillation is well within the kink length that is too small to be of concern. This challenge prevents the evaluation of $k_{\mathrm{I}}$ and $k_{\mathrm{II}}$ at zero or near zero kink length $(a=0)$ in Equations (65) and (66).

For cracks between similar materials, Equations (65) and (66) can be reduced to those of Cotterell and Rice [9] as

$$
\begin{aligned}
& k_{I}=\left(c_{R}+d_{R}\right) K_{I}^{A}-\left(c_{I}+d_{I}\right) K_{I I}^{A} \\
& k_{I I}=\left(c_{I}-d_{I}\right) K_{I}^{A}+\left(c_{R}-d_{R}\right) K_{I I}^{A}
\end{aligned}
$$

It should be noted that the stresses at the crack tip recover the traditional $1 / \sqrt{r}$ singularity in this case.

The local stress intensity factor solutions $k_{\mathrm{I}}$ and $k_{\mathrm{II}}$ for kinked cracks with finite kink lengths in lap-shear specimens can be expressed in the normalized forms based on the beam bending theory as

$$
\begin{aligned}
& k_{I}\left(\frac{a}{t_{u}}\right)=f_{I}\left(\frac{a}{t_{u}}\right) \cdot \frac{F}{b \sqrt{t_{u}}} \\
& k_{I I}\left(\frac{a}{t_{u}}\right)=f_{I I}\left(\frac{a}{t_{u}}\right) \cdot \frac{F}{b \sqrt{t}_{u}}
\end{aligned}
$$

and the local effective stress intensity factor solution $k_{e}$ can be expressed as

$$
k_{e}\left(\frac{a}{t_{u}}\right)=\sqrt{k_{I}^{2}\left(\frac{a}{t_{u}}\right)+k_{I I}^{2}\left(\frac{a}{t_{u}}\right)}=f_{e}\left(\frac{a}{t_{u}}\right) \cdot \frac{F}{b \sqrt{t_{u}}}
$$


where $f_{I}, f_{I I}$ and $f_{e}$ are dimensionless geometric functions which depend on the normalized kink length $a / t_{u}$ for this particular set of the geometry and material combination in the lap-shear specimen. The analytical local stress intensity factor $k_{I}$ and $k_{I I}$ solutions for the kinked cracks in the lap-shear specimens can be obtained using Equations (65) and (66) with the values of $K_{1}$ and $K_{2}$ obtained from the finite element analyses. The results will be presented in the normalized form in the following section. 


\section{Computational local stress intensity factor solutions for kinked cracks}

In this investigation, two-dimensional plane strain finite element analyses were conducted for the adhesive-bonded lap-shear specimen to investigate the local stress intensity factor solutions for kinked cracks with different kink lengths. It should be mentioned that the analytical local stress intensity factor solutions based on the global stress intensity factor solutions in Equations (65) and (66) are only valid when the kink length $a$ is small. Figure 6(a) shows a schematic of a two-dimensional finite element model of an adhesive-bonded lap-shear specimen with the boundary and loading conditions. The geometry, the loading and the boundary conditions of the specimen are the same as those shown in Figure 4(a). In Figure 6(a), a kinked crack is shown to grow from the right main crack tip into the upper magnesium sheet. Based on the micrograph in Figure 2(b), the kink angle is selected to be $30^{\circ}$. Figure 6(b) shows a close-up view of the finite element mesh near the kinked crack for $a / t_{u}=0.1$. The procedure of the finite element analyses is the same as that stated in section 4. Thirteen normalized kink lengths, $a / t_{u}=1.25 \times 10^{-5}, 3.125 \times 10^{-3}, 6.25 \times 10^{-3}, 0.0125,0.025,0.05,0.1,0.2,0.3,0.4,0.5$, 0.6 and 0.7 are used in this study. Note that the maximum normalized kink length observed in the experiment of Lai et al. [7] is about 0.7. Here, the local stress intensity factor solutions are obtained computationally for the adhesive-bonded lap-shear specimen with the material properties as specified in section 4 . The normalized local stress intensity factor solutions obtained from ABAQUS for these kink lengths are listed in Table 2. These computational results are then compared with the analytical solutions in Equations (65) and (66) based on the values of $K_{1}^{A}$ and $K_{2}^{A}$ obtained from the finite element analyses.

Figures 7(a) and 7(b) show the computational results and the analytical solutions for the dimensionless geometric function $f_{I}$ (normalized $k_{I}$ ) as a function of the normalized kink 
length $a / t_{u}$ in the linear and semi-log scales, respectively. As shown in Figure 7(b), the computational results approach to the analytical solution as the normalized kink length decreases to a very small value. Also, the computational local stress intensity factor $k_{I}$ increases significantly as the kink length increases while the analytical local stress intensity factor stays almost the same at large kink lengths.

Figures 7(c) and 7(d) show the computational results and the analytical solutions for the dimensionless geometric function $f_{I I}$ (normalized $k_{I I}$ ) as a function of the normalized kink length $a / t_{u}$ in the linear and semi-log scales, respectively. It is noted that the magnitude of the local stress intensity factor $k_{I I}$ is small compared to that of the local stress intensity factor $k_{I}$. As shown in Figure 7(d), the computational results approach to the analytical solution as the normalized kink length decreases to a very small value. Also, the computational local stress intensity factor $k_{I I}$ increases significantly as the kink length increases while the analytical local stress intensity factor just increases slightly at large kink lengths.

Figures 7(e) and 7(f) show the computational results and the analytical solutions for the dimensionless geometric function $f_{e}$ (normalized $k_{e}$ ) as a function of the normalized kink length $a / t_{u}$ in the linear and log-log scales, respectively. As shown in Figure 7(f), the computational results approach to the analytical solution as the kink length decreases. Also, the computational local stress intensity factor $k_{e}$ increases as the kink length increases while the analytical local stress intensity factor stays almost the same at large kink lengths. It is noted that both the computational results and the analytical solutions for $k_{e}$ are similar to those for $k_{I}$ because the magnitudes of the $k_{I I}$ solutions are small compared to those of the $k_{I}$ solutions. The 
results in Figure 7 indicate that the analytical solution may be used to estimate the effective local stress intensity factors when the kink length is very small.

For fatigue life estimation, the local effective stress intensity factor at zero or near zero kink length is needed for a kinked fatigue crack growth model. Based on the results presented earlier, a simple way to estimate the local stress intensity factor solutions at zero or near zero kink length in lap-shear specimen of dissimilar sheet materials under plane strain conditions is to choose a kink length that is reasonably small based on Equations (65) and (66). A suggested kink length is the average grain size. Using a kink length smaller than the average grain size for Equations (65) and (66) may not be practical since the material can no longer be treated as homogeneous linear elastic. The magnesium sheet used in this study contains highly deformed grain structure due to the rolling process. The microstructure consists of small broken grains and twins in larger grains. The grain size of the magnesium sheet ranges from 2 to $15 \mu \mathrm{m}$ with an average grain size of $5 \mu \mathrm{m}$ as reported in Santella et al. [1]. As shown in Figure 7(f), the effective stress intensity factor solution obtained from the analytical solution is $20 \%$ smaller than that obtained from the finite element analysis for the kink length equal to the average grain size of $5 \mu \mathrm{m}$. This value may be considered for the solution for zero or near zero kink length. The method provides an easy and yet reasonable approach for evaluating the stress intensity factor solutions for zero or near zero kink length and is useful for fatigue life estimations since the fatigue crack growth model needs the local stress intensity factors at zero or near zero kink length.

The increasing stress intensity factor solutions with the increasing kink length as shown from the results of the finite element analyses also explain the increasing kink length with the decreasing load range observed in the failed specimens under cyclic loading conditions. Since 
the stress intensity factors at the kinked crack tip increase as the kinked crack grows, it can eventually reach the critical fracture resistance of the magnesium sheet and causes the kinked crack to grow through the magnesium sheet. The maximum kink length calculated using the stress intensity factor solutions obtained from the finite element analyses and the fracture toughness of $K_{I c}=17.6 \mathrm{MPa} \sqrt{\mathrm{m}}$ for the $90^{\circ}$ specimens of the rolled magnesium AZ31 sheets in Somekawa and Mukai [20] are 0.91 and $1.37 \mathrm{~mm}$ at the load ranges of 4,630 and 2,900 N, respectively, for the kinked crack failure mode observed in the experiment. The critical maximum kink lengths observed in the magnesium sheets are 0.3 and $0.9 \mathrm{~mm}$ at the upper and lower bounds of the load ranges of 4,630 and 2,900 N, respectively. The general trends of the predicted and observed maximum kink lengths are in agreement. If the fracture toughness of $K_{I c}=10.8 \mathrm{MPa} \sqrt{\mathrm{m}}$ is selected as a fitting parameter, the predicted maximum kink lengths at the load ranges of 4,630 and 2,900 $\mathrm{N}$ are 0.91 and $1.37 \mathrm{~mm}$, respectively, which exactly match the experimental results. This suggests that the sharp turning of the kinked crack from $30^{\circ}$ to $90^{\circ}$ is due to reaching the critical fracture resistance of the magnesium sheet.

\section{Fatigue Life Estimation}

The fatigue life estimations for the lap-shear specimens are based on the Paris law for crack growth and the stress intensity factor solutions obtained from the finite element analyses. For the kinked crack failures in the lap-shear specimens, the fatigue crack growth is under local combined mode I and mode II loading conditions. An equivalent stress intensity factor $k_{e q}$ is defined in Broek [21] as

$k_{e q}(a)=\sqrt{k_{I}(a)^{2}+\gamma k_{I I}(a)^{2}}$ 
where $\gamma$ is an empirical constant to account for the sensitivity of materials to the mode II loading conditions. For lack of any further information, $\gamma$ is assumed to be 1 in this paper. Now the Paris law is used to describe the fatigue crack growth for the kinked crack as

$$
\frac{\mathrm{d} a}{\mathrm{~d} N}=C\left(\Delta k_{e q}(a)\right)^{m}
$$

where $N$ is the life or number of cycles, $C$ and $m$ are the material constants, and $\Delta k_{e q}$ is the range of the equivalent stress intensity factor as a function of the kink length $a$.

Since the local stress intensity factors are functions of the kink length $a$, the fatigue life of the lap-shear specimens can be obtained by integrating Equation (73) as

$$
N=\frac{1}{C}\left\{\int_{0}^{a_{1}}\left[\Delta k_{e q}(a)\right]^{-m} \mathrm{~d} a+\int_{a_{1}}^{a_{2}}\left[\Delta k_{e q}(a)\right]^{-m} \mathrm{~d} a+\cdots+\int_{a_{i-1}}^{a_{i}}\left[\Delta k_{e q}(a)\right]^{-m} \mathrm{~d} a\right\}
$$

where $a_{1}, a_{2}, \ldots, a_{i-1}$ and $a_{i}$ represent the values of different kink lengths. Here, $a_{i}$ represents the crack length when the maximum equivalent or effective stress intensity factor reaches the fracture toughness value of 17.6 MPa $\sqrt{\mathrm{m}}$ (for the $90^{\circ}$ specimens in Somekawa and Mukai [20]). The stress intensity factor solutions for different kink lengths obtained from finite element analyses were used to estimate the fatigue lives. Since the material constants for the Paris law for the rolled AZ31B-H24 used in this study are not available, the material constants were chosen from the AZ31B with a similar grain size and load ratio. The values of $C$ and $m$ used to estimate the fatigue life are $4 \times 10^{-7} \mathrm{~mm} / \mathrm{cycle} /(\mathrm{MPa} \sqrt{\mathrm{m}})^{n}$ and 2.7 , respectively, as presented in Ishihara et al. [22]. It should be noted that the fatigue crack growth rate varies with the grain size, load ratio, texture and humidity for AZ31B. Uematsu et al. [23] studied the fatigue crack growth rate for extruded AZ31B and found that the fatigue crack growth rate decreases with the decreasing grain size. Zheng et al. [24] conducted tests to obtain the fatigue crack growth rates 
under different load ratios for extruded AZ31B and found that the fatigue crack growth rate increases with the increasing load ratio. Ishihara et al. [25] studied the fatigue crack growth rates for extruded and rolled AZ31 and found a significant texture effect on the fatigue crack growth rate for both extruded and rolled AZ31. Tokaji et al. [26] conducted the fatigue tests under dry and humid air conditions for rolled AZ31 and found an order of magnitude difference in fatigue crack growth rate.

Figure 8 shows the experimental results and fatigue life estimations for adhesive-bonded lap-shear specimens of magnesium and steel sheets. Under low-cycle loading conditions, the crack grew in an interfacial failure mode between the epoxy layer and the steel sheet during the initial load. As cyclic loads continued, the interfacial failure gradually changed to near interface cohesive failure in the epoxy layer. Under high-cycle loading conditions, the specimens failed in the kinked fatigue crack growth mode in the magnesium sheet. The estimated fatigue lives for the kinked crack failure mode for the adhesive-bonded lap-shear specimens are shown as the dash line in Figure 8. It appears that the fatigue life estimations are lower than the experimental results. However, the trend of the fatigue life estimations in general agrees well with that of the experimental results. It is noted that the values of $C$ and $m$ used to estimate the fatigue lives in this study are from an extruded AZ31. The distinct texture feature in the rolled AZ31 compared to that of the extruded AZ31 can change the values of $C$ and $m$ and, in turn, change the corresponding fatigue life estimations. It should be mentioned that the computational results of the local stress intensity factor solutions at the kinked crack length of $5 \mu \mathrm{m}$ was used for the fatigue life estimation from the crack length of $0 \mu \mathrm{m}$ to $5 \mu \mathrm{m}$. When the computational stress intensity factor solutions are not available, the analytical solution at the kinked crack length of 5 $\mu \mathrm{m}$ may be used for fatigue life estimation in this crack length range. 
Based on the linear elastic fracture mechanics, the plastic zone size of the kinked crack in the magnesium sheet under cyclic loading conditions at the time when the effective local stress intensity factor reaches the fracture toughness of $17.6 \mathrm{MPa} \sqrt{\mathrm{m}}$ (or $10.8 \mathrm{MPa} \sqrt{\mathrm{m}}$ ) are small compared to the crack lengths of interest. Therefore, the use of linear elastic fracture mechanics and the kinked crack growth model for the fatigue life estimations is reasonable in this study. Based on the beam bending theory, the magnesium sheet is yielded substantially at the maximum load of 5,144 $\mathrm{N}$ for the load range of 4,630 $\mathrm{N}$ (the largest load range that resulted in the kinked crack failure mode) due to the combined tension and bending. However, the magnesium sheet near the right main crack tip was under elastic loading and unloading condition after the initial loading since the stress range for elastic loading and unloading is quite large due to the large yield stress of AZ31. The plastic flow near the crack tip is due to the singular behavior near the crack tip. Therefore, the fatigue life estimations based on the kinked crack growth model is reasonable with the stress intensity factor solutions.

\section{Conclusions}

In this paper, stress intensity factor solutions for adhesive-bonded lap-shear specimens of magnesium alloy AZ31 and hot-dip-galvanized (HDG) mild steel sheets with and without kinked cracks are investigated for fatigue life estimations. First, the kinked fatigue crack failure mode of the adhesive-bonded lap-shear specimens is briefly reviewed. Then, the analytical global $J$ integral and effective stress intensity factor solutions for main cracks in lap-shear specimens of three dissimilar sheets under plane strain conditions are developed based on the beam bending theory. The global effective stress intensity factor solutions for the main cracks in 
the lap-shear specimens from the corresponding finite element analyses are then presented and validated by the analytical solutions.

Next, the local stress intensity factor solutions for kinked cracks with the experimentally observed kink angle as functions of the kink length from the corresponding finite element analyses are presented and the computational solutions are also compared with the analytical solutions at small kink lengths. The results indicate that the computational local stress intensity factor solutions for kinked cracks approach to the analytical solutions as the kink length decreases to a very small value and the kinked crack is under dominant mode I loading conditions. The computational results also indicate that the local stress intensity factor solutions at a small kink length of microstructural significance may be used as the stress intensity factor solutions for zero or near zero kink length for fatigue life estimations when the computational results are not available.

Finally, the computational local stress intensity factor solutions are adopted to estimate the fatigue lives of the lap-shear specimens based on a kinked crack growth model and available material constants for the Paris law. The fatigue life estimations are lower than the experimental results. However, the general trend of fatigue life estimations agrees with that of the experimental results.

\section{Acknowledgements}

This research was sponsored by the U.S. Department of Energy, Assistant Secretary for Energy Efficiency and Renewable Energy, Office of Vehicle Technologies, as part of the Lightweight Materials Program. Helpful discussion with Drs. Tsung-Yu Pan, Michael Santella 
and Zhili Feng of Oak Ridge National Laboratory and Dr. Teresa Rinker of General Motors are greatly appreciated.

\section{References}

[1] Santella M, Franklin T, Pan J, Pan T-Y, Brown E. Ultrasonic spot welding of AZ31B to galvanized mild Steel. SAE International Journal of Materials and Manufacturing. 2010;3:652-7.

[2] Hetrick E, Jahn R, Reatherford L. Ultrasonic spot welding: a new tool for aluminum joining. Welding Journal. 2005;84:26-30.

[3] Jahn R, Cooper R, Wilkosz D. The effect of anvil geometry and welding energy on microstructures in ultrasonic spot welds of AA6111-T4. Metallurgical and Materials Transactions A: Physical Metallurgy and Materials Science. 2007;38:570-83.

[4] Wright NW, Robson JD, Prangnell PB. Effects of thickness combinations on joint properties and process windows in ultrasonic metal welding. SAE technical paper no. 2009-01-0027. Warrendale, PA: Society of Automotive Engineers; 2009.

[5] Watanabe A, Watanabe T, Sasaki T, University N. Ultrasonic welding mild steel sheet to almg alloy sheet. Advanced Materials Research. 2010;89-91:627-32.

[6] Franklin T, Pan J, Santella M, Pan T-Y. Fatigue behavior of dissimilar ultrasonic spot welds in lap-shear specimens of magnesium and steel sheets. SAE International Journal of Materials and Manufacturing. 2011;4:581-8.

[7] Lai W-J, Pan J, Feng Z, Santella M, Pan T-Y. Failure mode and fatigue behavior of ultrasonic spot welds with adhesive in lap-shear specimens of magnesium and steel sheets. SAE International Journal of Materials and Manufacturing. 2013;6:279-85.

[8] Franklin T. Fatigue behavior and life estimation for dissimilar ultrasonic welds in lap-shear specimens of magnesium and galvanized steel sheets [dissertation]. Ann Arbor (MI): University of Michigan; 2013.

[9] Cotterell B, Rice JR. Slightly curved or kinked cracks. International Journal of Fracture. 1980;16:155-69.

[10] He M-Y, Hutchinson JW. Kinking of a crack out of an interface. Journal of Applied Mechanics. 1989;56:270-8.

[11] Zhang S. Stress intensities derived from stresses around a spot weld. International Journal of Fracture. 1999;99:239-57.

[12] Rice JR. A path independent integral and the approximate analysis of strain concentration by notches and cracks. Journal of Applied Mechanics. 1968;35:379-86. 
[13] Wang D-A, Lin P-C, Pan J. Geometric functions of stress intensity factor solutions for spot welds in lap-shear specimens. International Journal of Solids and Structures. 2005;42:6299-318.

[14] Wang D-A, Lin S-H, Pan J. Stress intensity factors for spot welds and associated kinked cracks in cup specimens. International Journal of Fatigue. 2005;27:581-98.

[15] Lin P-C, Pan J. Closed-form structural stress and stress intensity factor solutions for spot welds in commonly used specimens. Engineering Fracture Mechanics. 2008;75:5187-206.

[16] Rice JR, Sih GC. Plane problems of cracks in dissimilar media. Journal of Applied Mechanics. 1965;32:418-23.

[17] Rice JR. Elastic fracture mechanics concepts for interfacial cracks. Journal of Applied Mechanics. 1988;55:98-103.

[18] ABAQUS v6.12 user manual. Providence, RI: SIMULIA; 2012.

[19] He M-Y, Hutchinson JW. Kinking of a crack out of an interface: tabulated solution coefficients. Harvard University Report MECH-113A. 1988.

[20] Somekawa H, Mukai T. Fracture toughness in a rolled AZ31 magnesium alloy. Journal of Alloys and Compounds. 2006;417:209-13.

[21] Broek D. Elementary engineering fracture mechanics. 4th ed. Martinus Nijhoff Publisher; 1986.

[22] Ishihara S, McEvily AJ, Sato M, Taniguchi K, Goshima T. The effect of load ratio on fatigue life and crack propagation behavior of an extruded magnesium alloy. International Journal of Fatigue. 2009;31:1788-94.

[23] Uematsu Y, Tokaji K, Kamakura M, Uchida K, Shibata H, Bekku N. Effect of extrusion conditions on grain refinement and fatigue behaviour in magnesium alloys. Materials Science and Engineering: A. 2006;434:131-40.

[24] Zheng S, Yu Q, Jiang Y. An experimental study of fatigue crack propagation in extruded AZ31B magnesium alloy. International Journal of Fatigue. 2013;47:174-83.

[25] Ishihara S, Taneguchi S, Shibata H, Goshima T, Saiki A. Anisotropy of the fatigue behavior of extruded and rolled magnesium alloys. International Journal of Fatigue. 2013;50:94-100.

[26] Tokaji K, Nakajima M, Uematsu Y. Fatigue crack propagation and fracture mechanisms of wrought magnesium alloys in different environments. International Journal of Fatigue.

2009;31:1137-43. 
Table 1. The dimensionless geometric functions or normalized stress intensity factor solutions obtained from ABAQUS and the analytical solution in Equation (53) for the main cracks.

\begin{tabular}{|l|c|c|}
\hline & Left & Right \\
\hline$F_{1}$ (computation) & 0.361 & 0.288 \\
\hline$F_{2}$ (computation) & 0.511 & 0.778 \\
\hline$F_{e}$ (computation) & 0.625 & 0.830 \\
\hline$F_{e}$ (analytical solution) & 0.622 & 0.828 \\
\hline$F_{2} / F_{1}$ (computation) & 1.413 & 2.702 \\
\hline
\end{tabular}


Table 2. The normalized local stress intensity factor solutions obtained from ABAQUS for different kink lengths at a kink angle of $\varphi=30^{\circ}$.

\begin{tabular}{|c|c|c|c|c|c|c|}
\hline$a / t_{u}$ & $1.25 \times 10^{-5}$ & $3.125 \times 10^{-3}$ & $6.25 \times 10^{-3}$ & 0.0125 & 0.025 & 0.05 \\
\hline$f_{I}$ & 1.346 & 1.486 & 1.529 & 1.590 & 1.680 & 1.808 \\
\hline$f_{I I}$ & -0.241 & 0.073 & 0.146 & 0.243 & 0.372 & 0.545 \\
\hline$f_{e}$ & 1.368 & 1.488 & 1.536 & 1.609 & 1.720 & 1.888 \\
\hline
\end{tabular}

Table 2 continued

\begin{tabular}{|c|c|c|c|c|c|c|c|}
\hline$a / t_{u}$ & 0.1 & 0.2 & 0.3 & 0.4 & 0.5 & 0.6 & 0.7 \\
\hline$f_{I}$ & 1.999 & 2.312 & 2.618 & 2.956 & 3.346 & 3.810 & 4.368 \\
\hline$f_{I I}$ & 0.767 & 1.066 & 1.300 & 1.520 & 1.750 & 2.004 & 2.299 \\
\hline$f_{e}$ & 2.141 & 2.546 & 2.923 & 3.324 & 3.776 & 4.305 & 4.936 \\
\hline
\end{tabular}




\section{Figure captions}

Figure 1. (a) A top view and (b) a bottom view of an adhesive-bonded lap-shear specimen. (c) A schematic of an adhesive-bonded lap-shear specimen. The applied force $F$ is shown as the bold arrows.

Figure 2. (a) An optical micrograph of a failed specimen at the fatigue life of $3.6 \times 10^{4}$ cycles under a load range of 4,570 N. (b) A close-up view of the kinked crack growing into the upper magnesium sheet at an angle of about $30^{\circ}$.

Figure 3. (a) A schematic of the right crack tip in an adhesive-bonded lap-shear specimen showing the normal stresses $\sigma_{u i}{ }_{2} \sigma_{u o}{ }_{2} \sigma_{l 1 i}, \sigma_{l 1 o}, \sigma_{l 2 i}$ and $\sigma_{l 2 o}$ at the inner $(i)$ and outer $(o)$ surfaces of the upper sheet $(u)$, lower sheet $1(l 1)$ and lower sheet $2(l 2)$, respectively. (b) A schematic of an adhesive-bonded lap-shear specimen near the crack tips showing the positions of

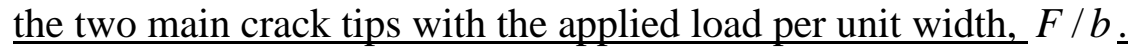

Figure 4. (a) A schematic of a two-dimensional adhesive-bonded lap-shear specimen with the boundary and loading conditions. Close-up views of the finite element meshes near (b) the right crack tip and (c) the left crack tip.

Figure 5. A schematic of a main crack and a kinked crack with the kink length $a$ and the kink angle $\varphi$.

Figure 6. (a) A schematic of a two-dimensional finite element model of an adhesive-bonded lapshear specimen with a kinked crack with the boundary and loading conditions. (b) A close-up view of the finite element mesh near the right crack tip showing the preformed crack and the kinked crack.

Figure 7. The computational results and analytical solutions for (a) $f_{I}$ in the linear scale, (b) $f_{I}$ in the semi-log scale, (c) $f_{\text {II }}$ in the linear scale, (d) $f_{\text {II }}$ in the semi-log scale, (e) $f_{e}$ in the linear scale, and (f) $f_{e}$ in the log-log scale as a function of the normalized kink length $a / t_{u}$ for $\varphi=30^{\circ}$ for the adhesive-bonded lap-shear specimen.

Figure 8. Experimental results of the fatigue tests at the load ratio $\mathrm{R}$ of 0.1 and fatigue life estimations for the adhesive-bonded lap-shear specimens. 


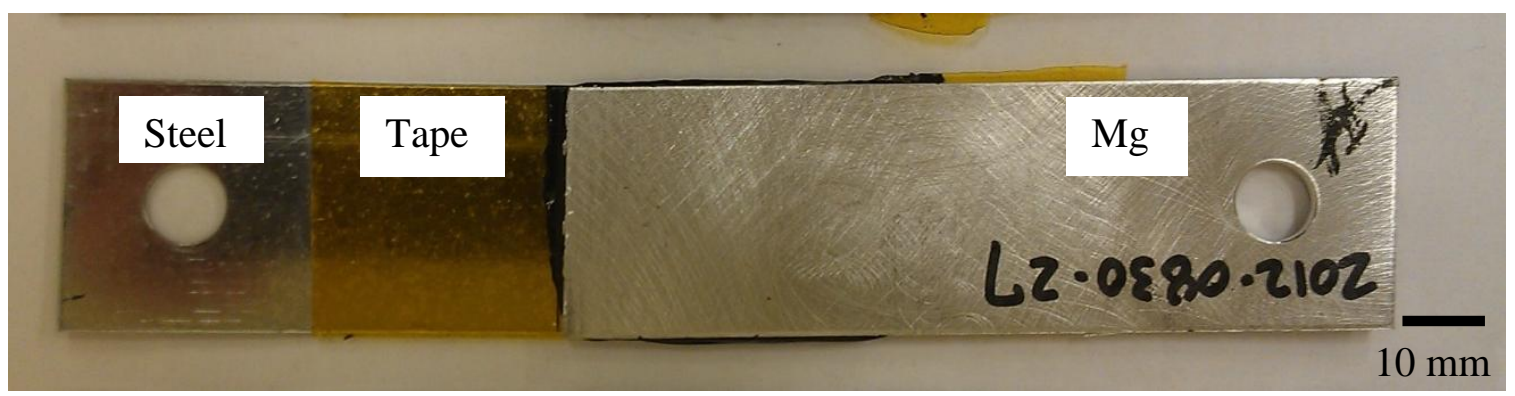

(a)

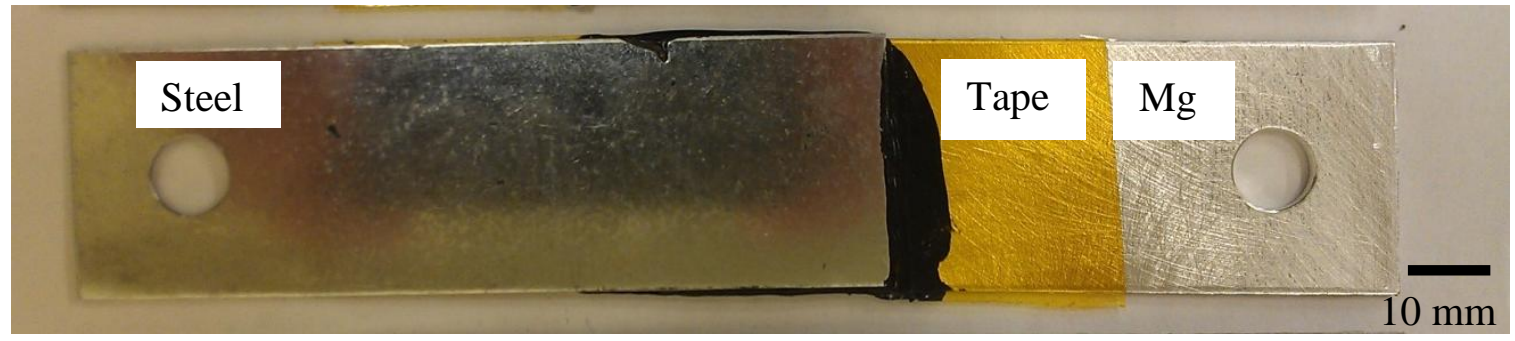

(b)

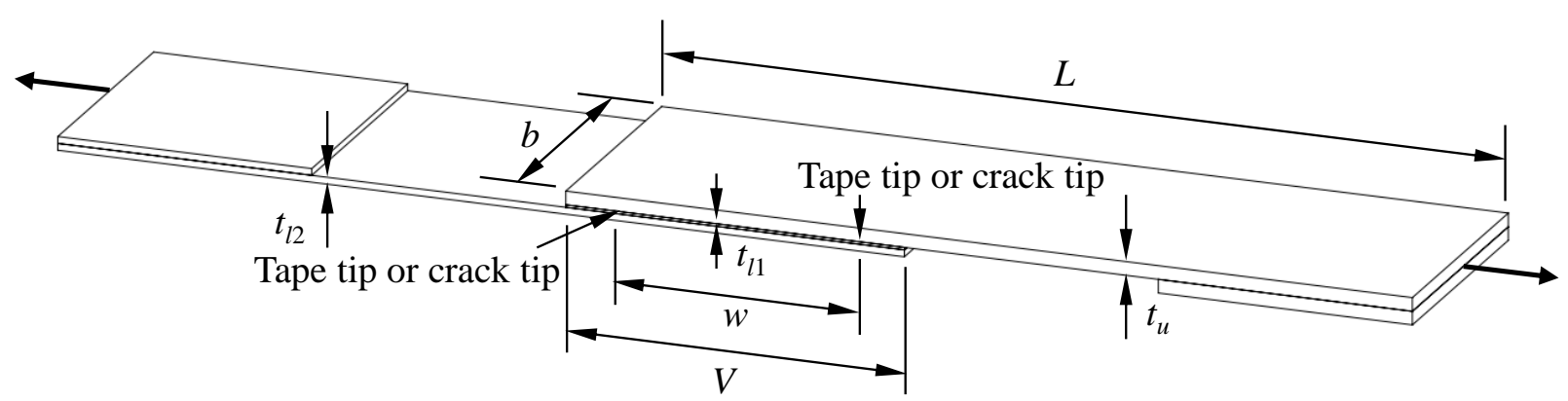

(c)

Figure 1. (a) A top view and (b) a bottom view of an adhesive-bonded lap-shear specimen. (c) A schematic of an adhesive-bonded lap-shear specimen. The applied force $F$ is shown as the bold arrows. 


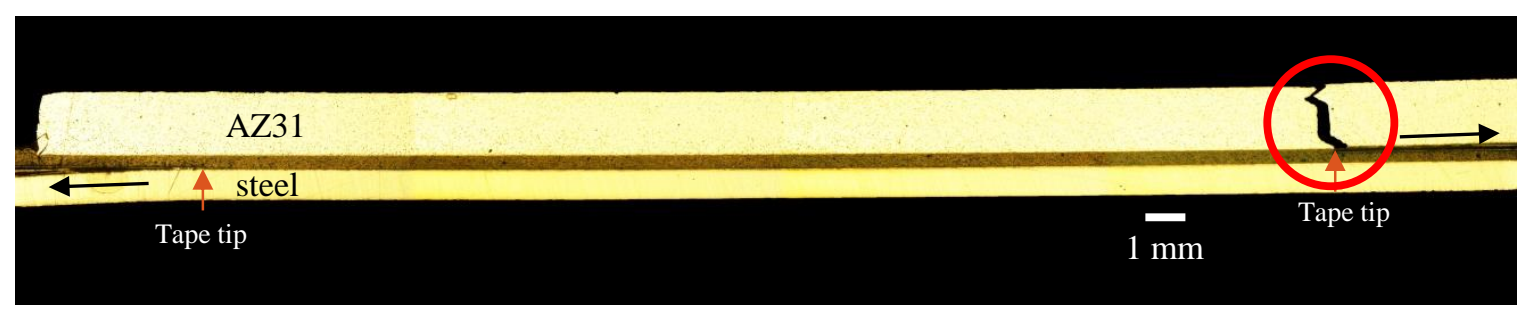

(a)

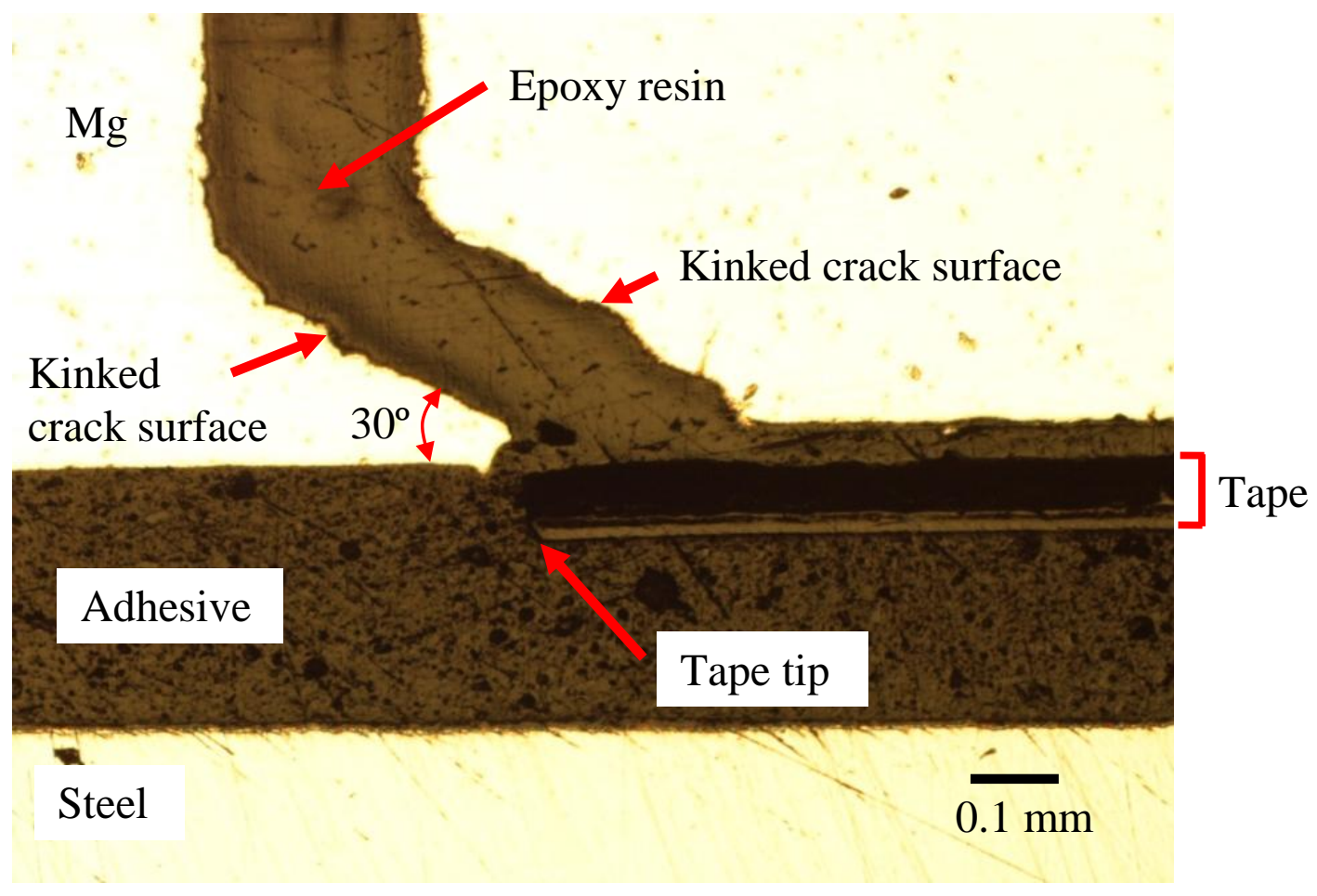

(b)

Figure 2. (a) An optical micrograph of a failed specimen at the fatigue life of $3.6 \times 10^{4}$ cycles under a load range of 4,570 N. (b) A close-up view of the kinked crack growing into the upper magnesium sheet at an angle of about $30^{\circ}$. 


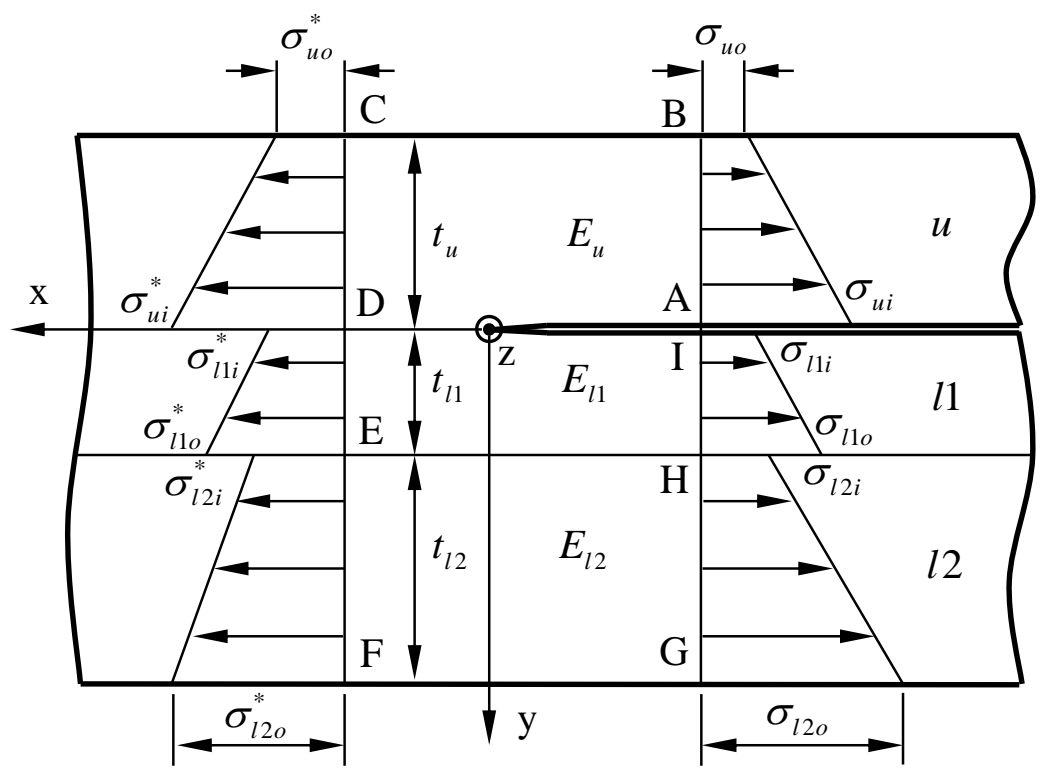

(a)

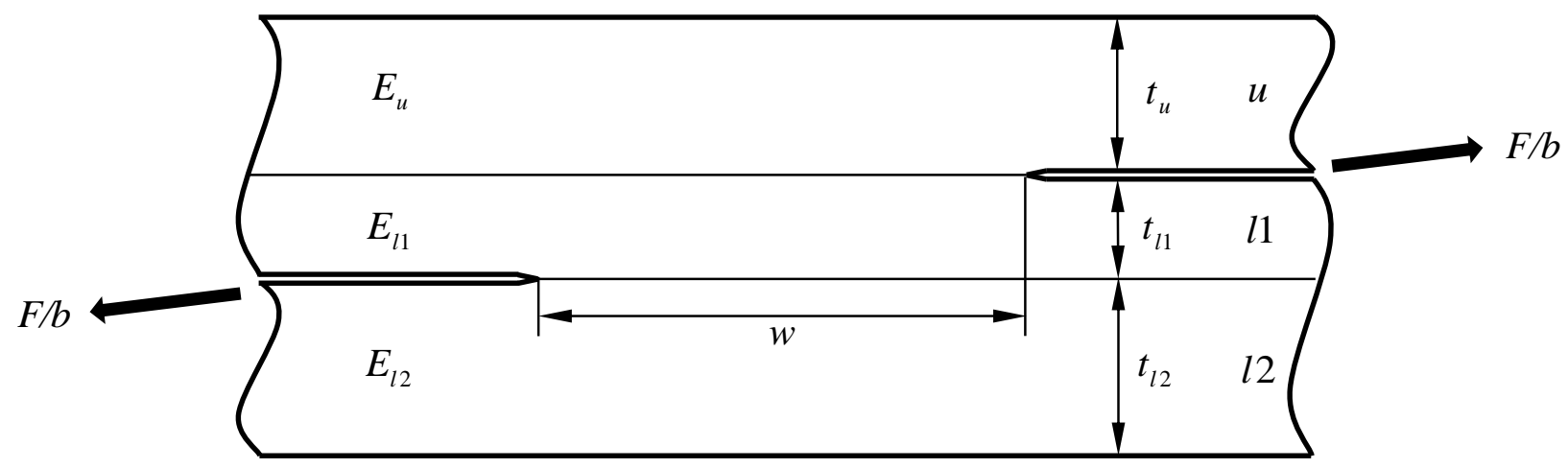

(b)

Figure 3. (a) A schematic of the right crack tip in an adhesive-bonded lap-shear specimen showing the normal stresses $\sigma_{u i}, \sigma_{u o}, \sigma_{l 1 i}, \sigma_{l 1 o}, \sigma_{l 2 i}$ and $\sigma_{l 2 o}$ at the inner $(i)$ and outer $(o$ ) surfaces of the upper sheet $(u)$, lower sheet 1 ( $l 1)$ and lower sheet 2 (l2), respectively. (b) A schematic of an adhesive-bonded lap-shear specimen near the crack tips showing the positions of the two main crack tips with the applied load per unit width, $F / b$. 


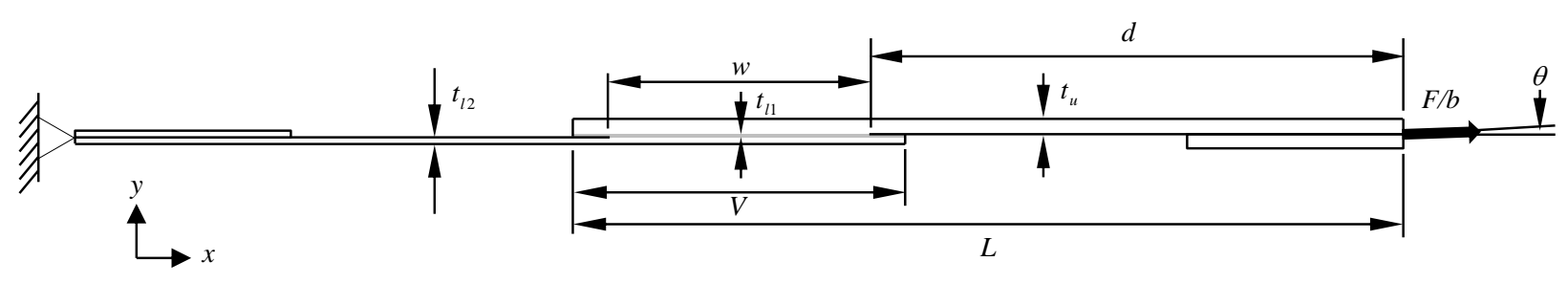

(a)

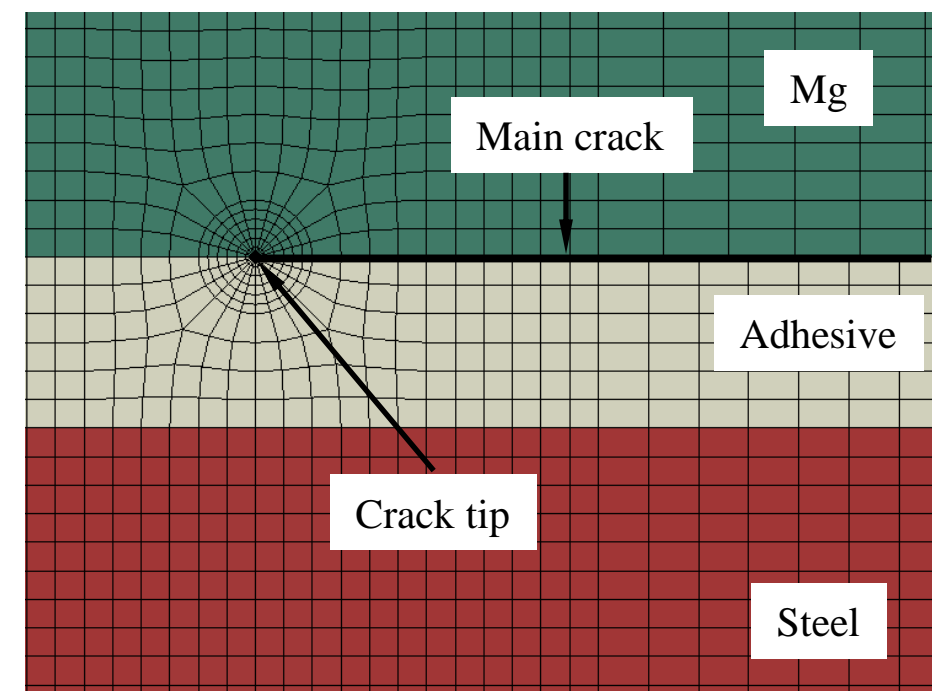

(b)

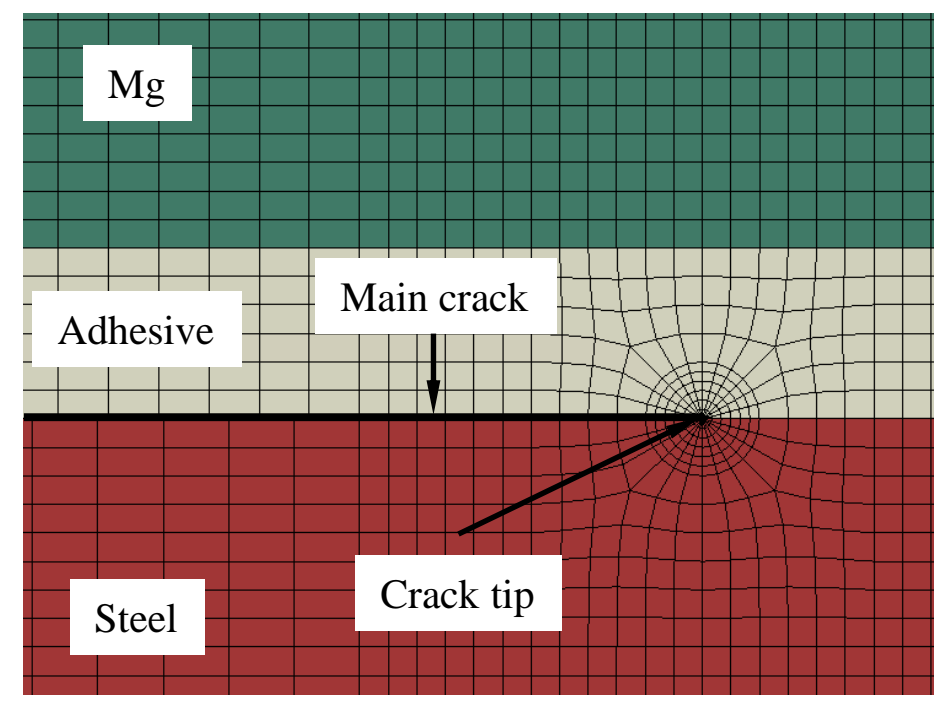

(c)

Figure 4. (a) A schematic of a two-dimensional adhesive-bonded lap-shear specimen with the boundary and loading conditions. Close-up views of the finite element meshes near (b) the right crack tip and (c) the left crack tip. 


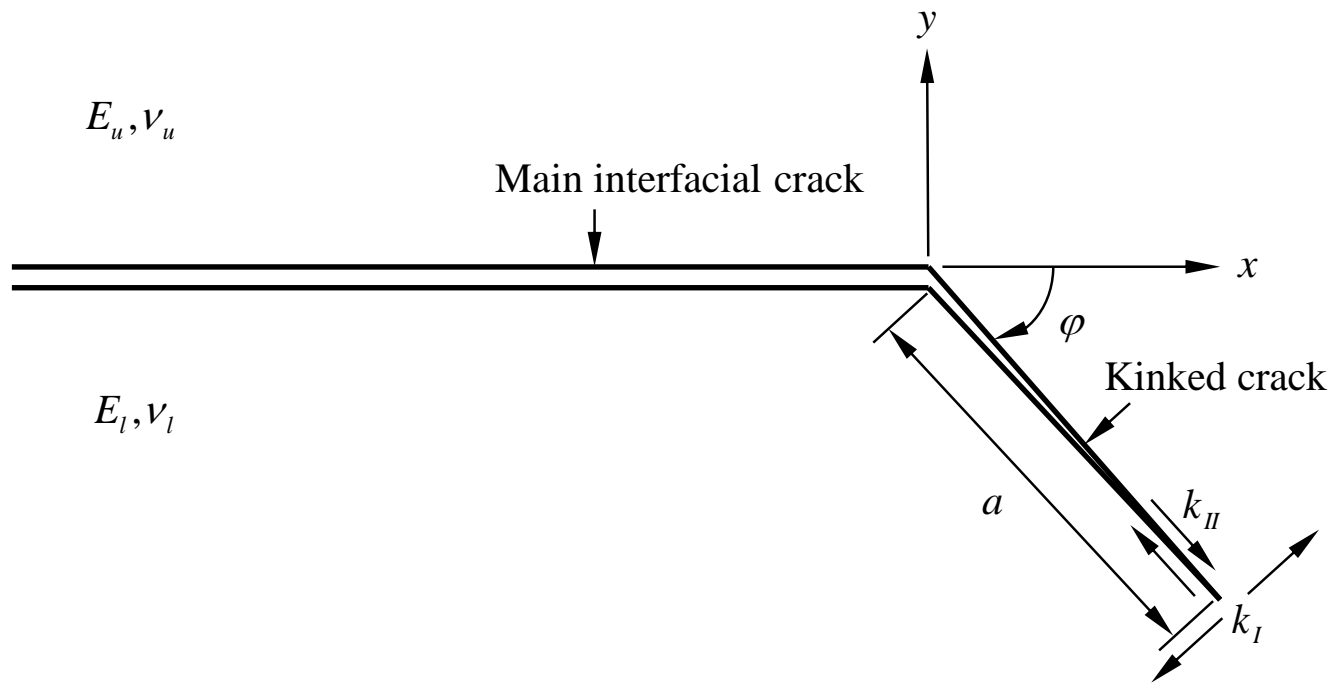

Figure 5. A schematic of a main crack and a kinked crack with the kink length $a$ and the kink angle $\varphi$. 


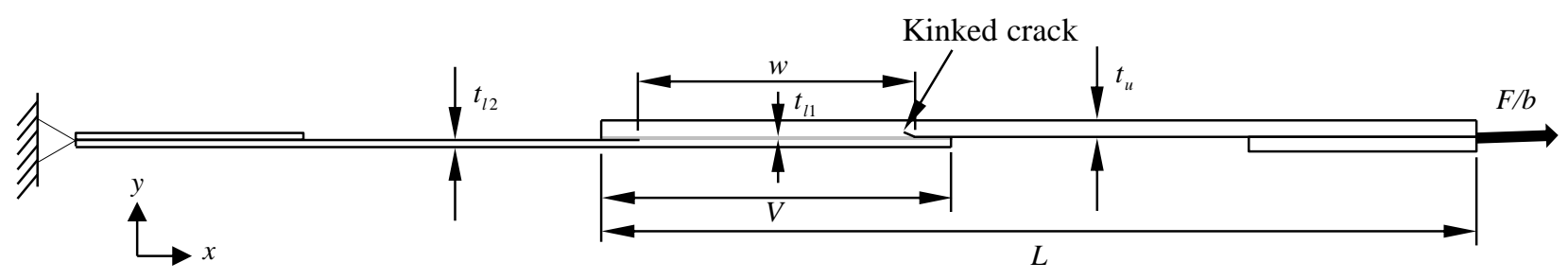

(a)

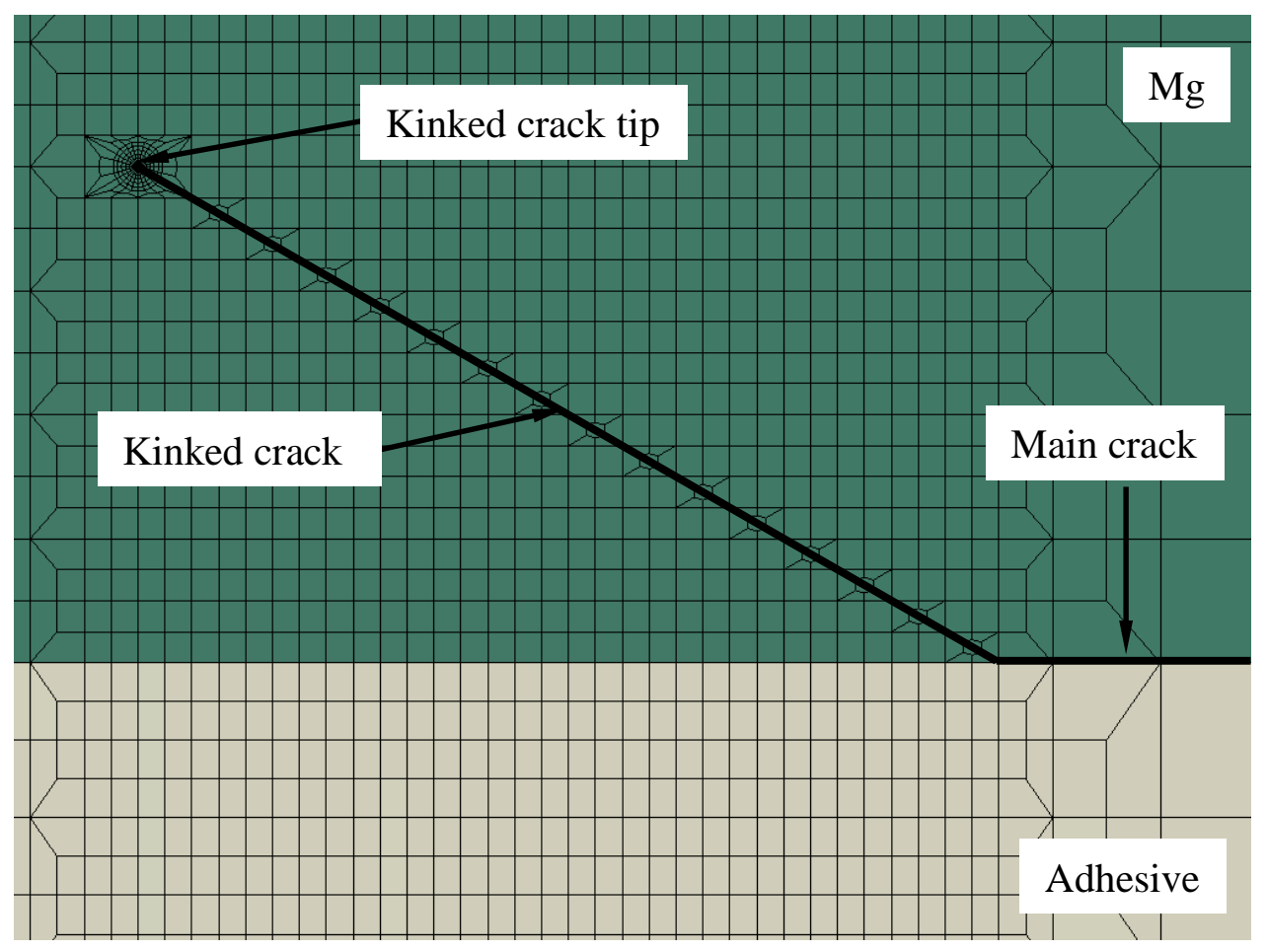

(b)

Figure 6. (a) A schematic of a two-dimensional finite element model of an adhesive-bonded lapshear specimen with a kinked crack with the boundary and loading conditions. (b) A close-up view of the finite element mesh near the right crack tip showing the preformed crack and the kinked crack. 


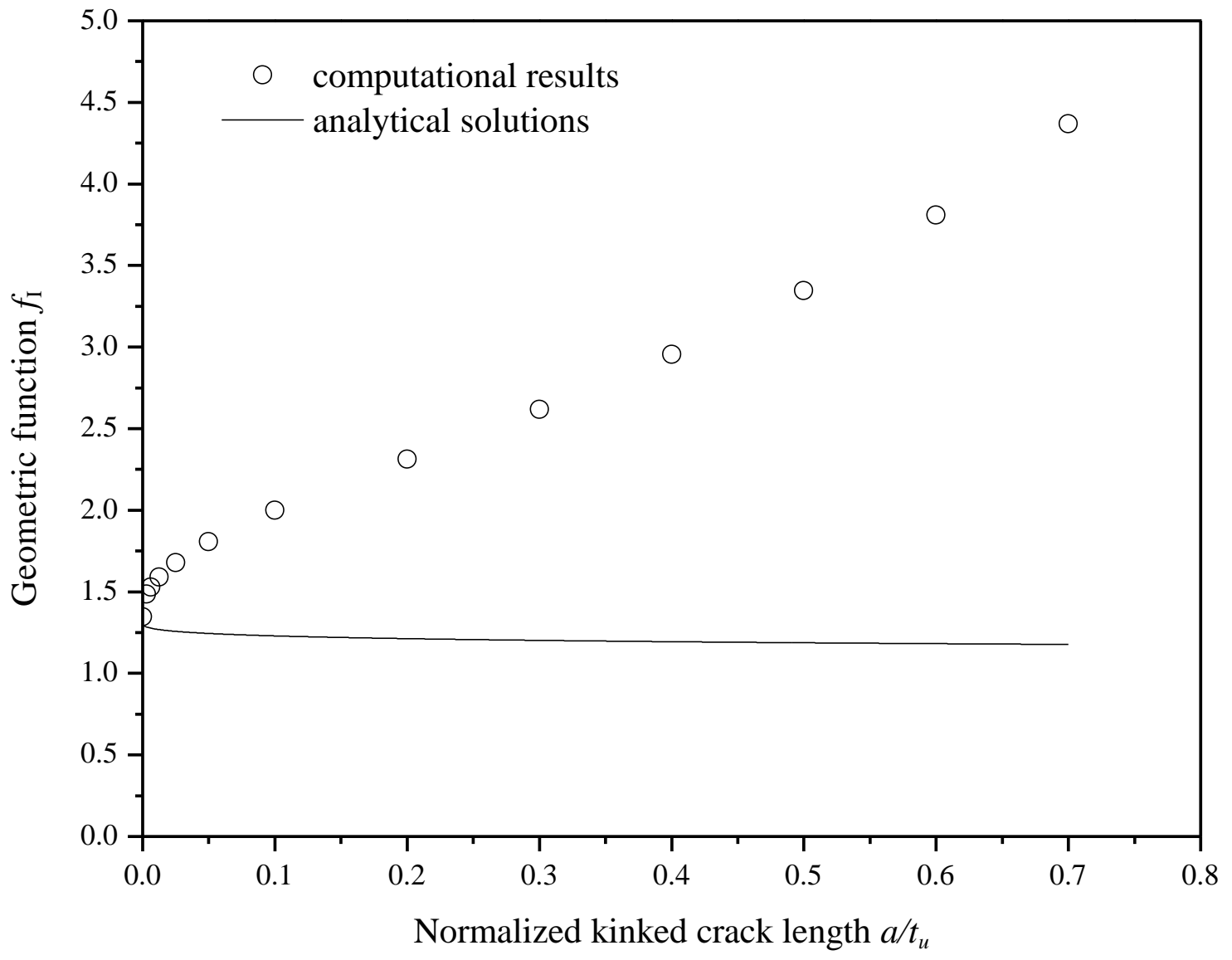

(a) 


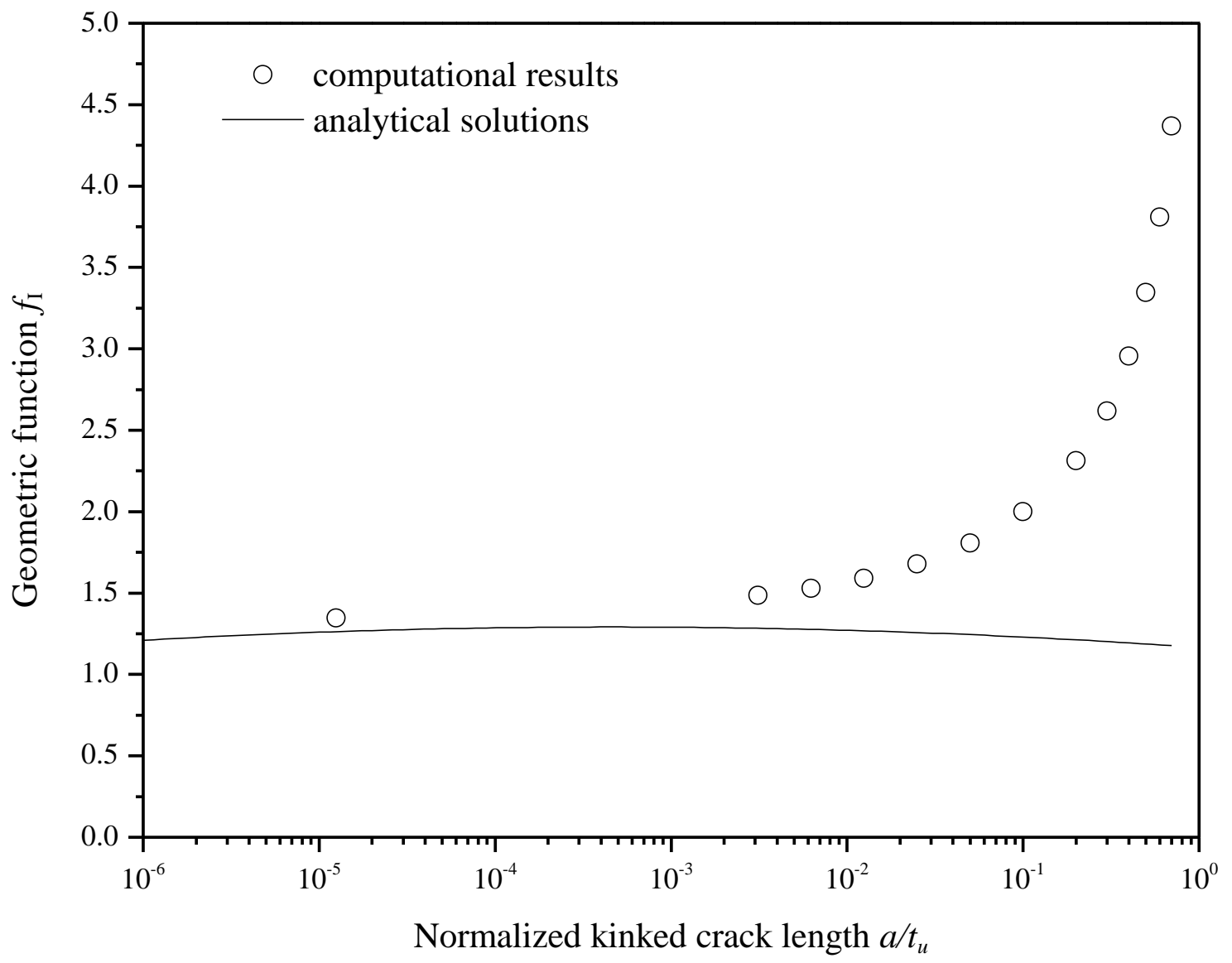

(b) 


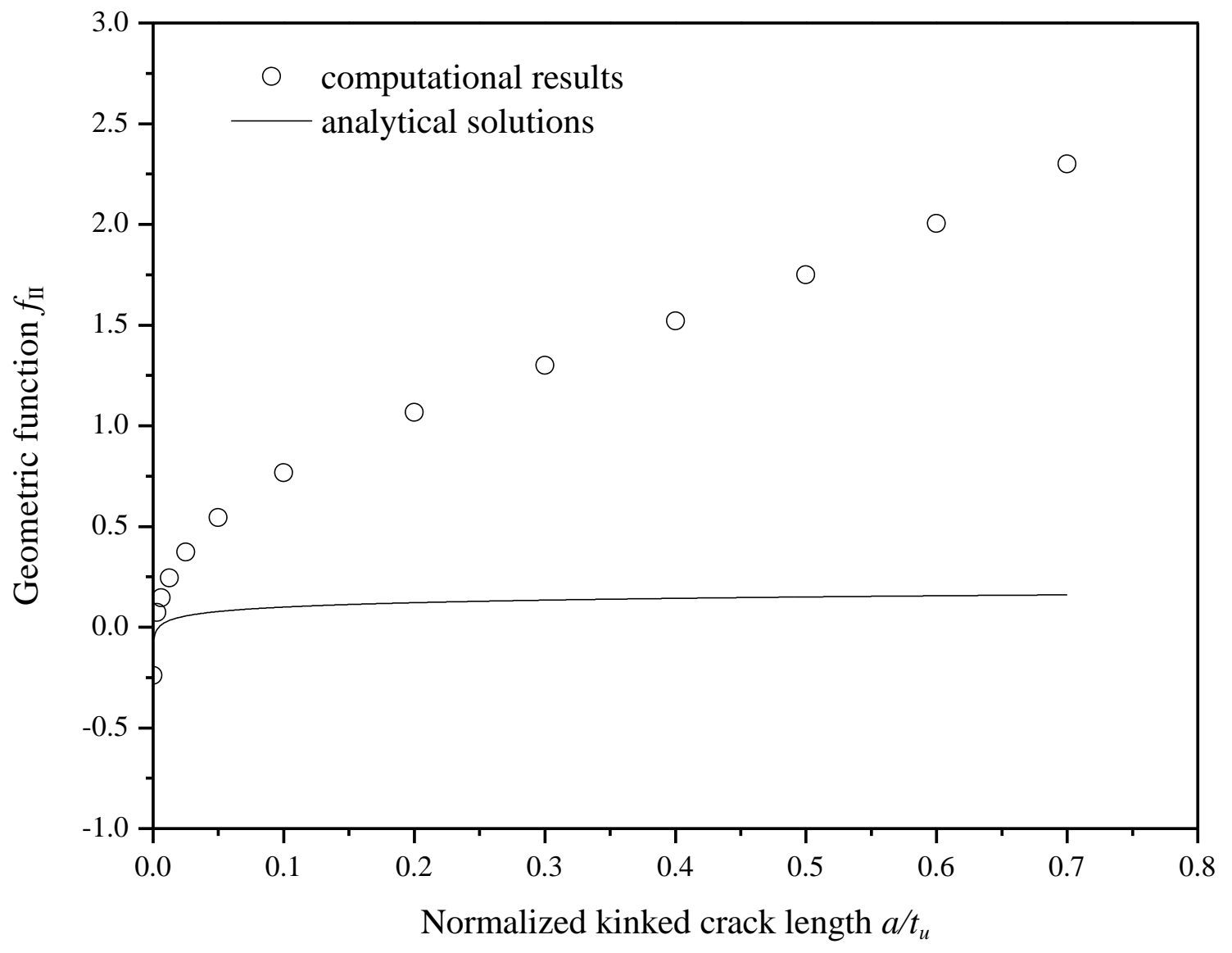

(c) 


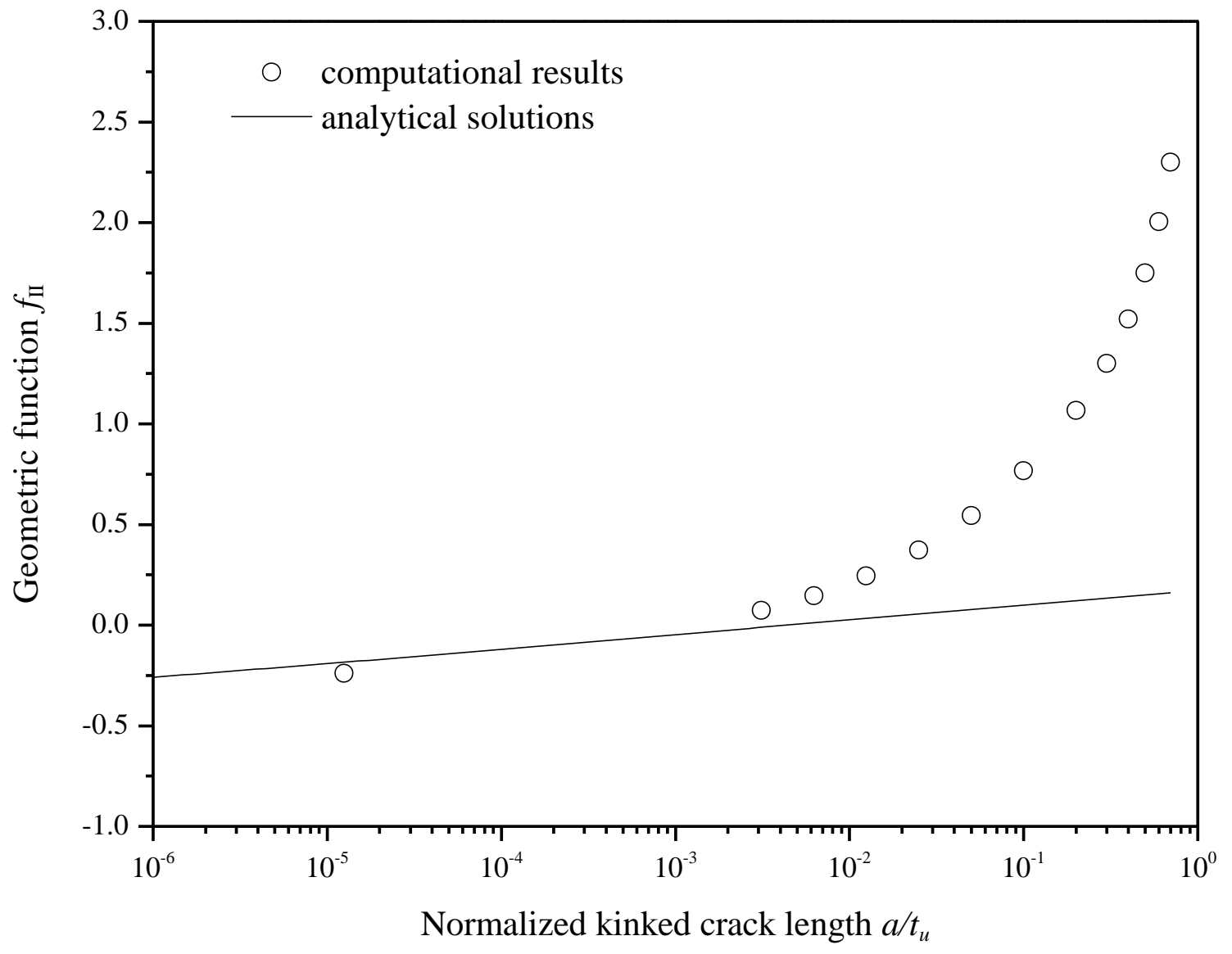

(d) 


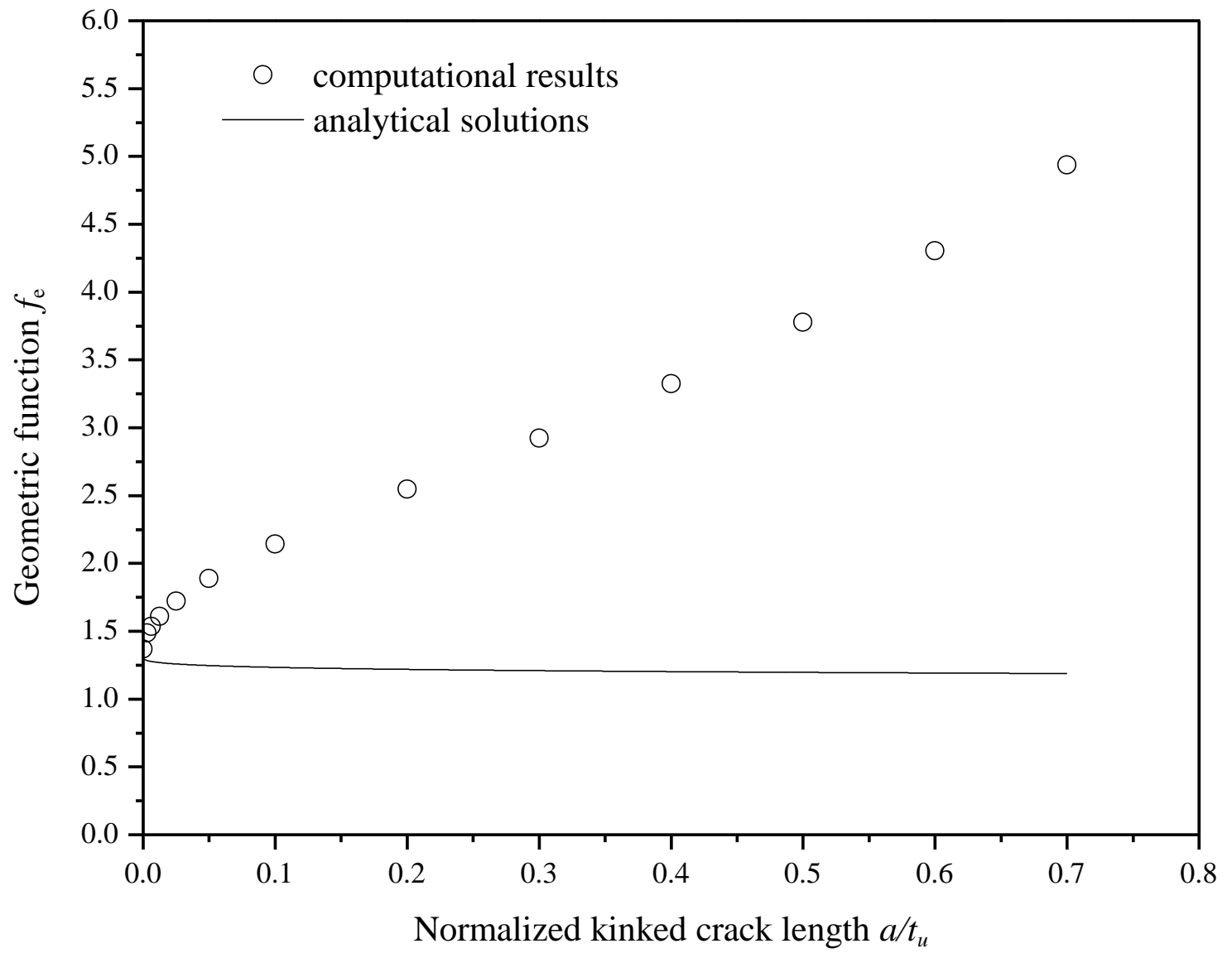

(e) 


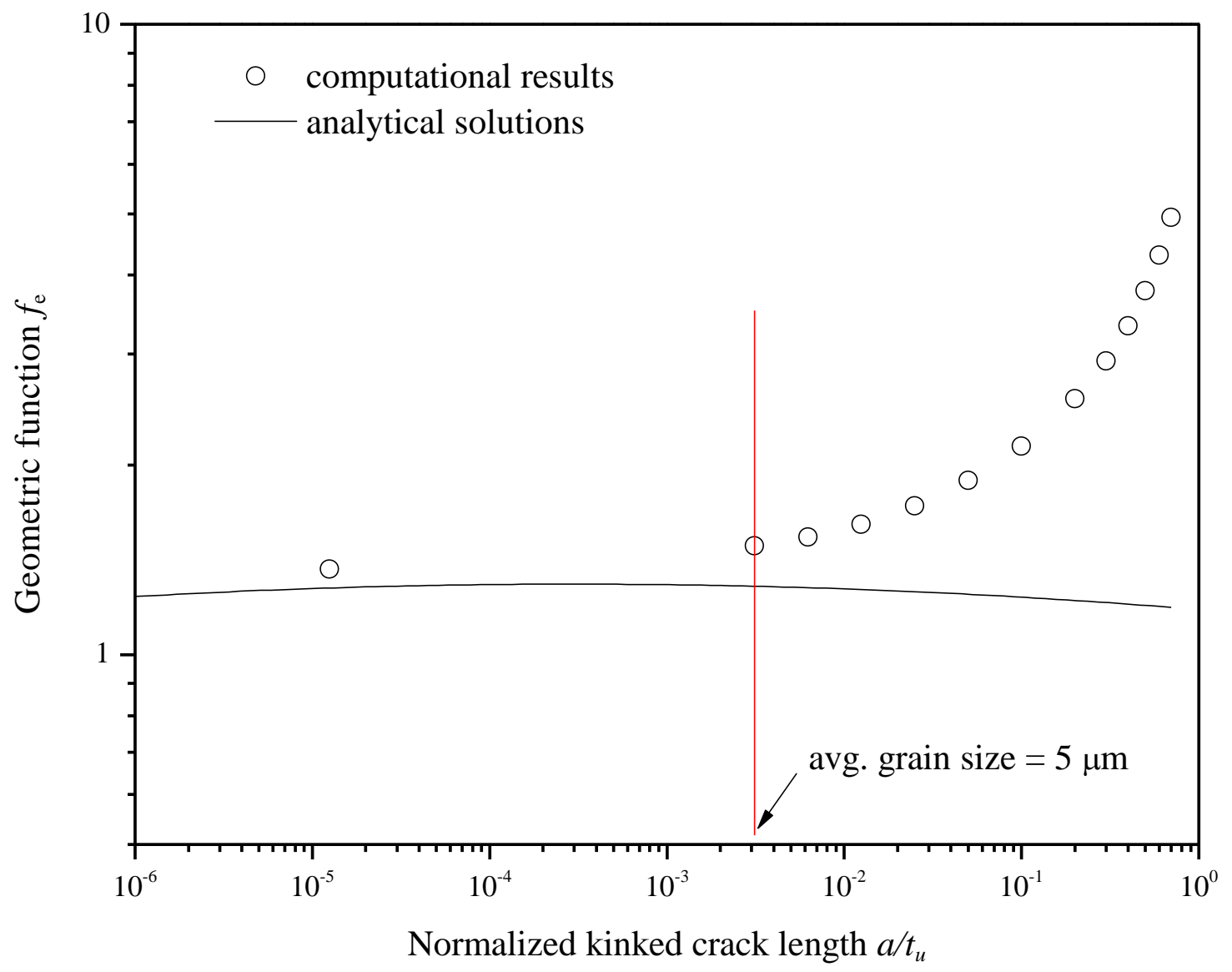

(f)

Figure 7. The computational results and analytical solutions for (a) $f_{I}$ in the linear scale, (b) $f_{I}$ in the semi-log scale, (c) $f_{I I}$ in the linear scale, (d) $f_{I I}$ in the semi-log scale, (e) $f_{e}$ in the linear scale, and (f) $f_{e}$ in the log-log scale as a function of the normalized kink length $a / t_{u}$ for $\varphi=30^{\circ}$ for the adhesive-bonded lap-shear specimen. 


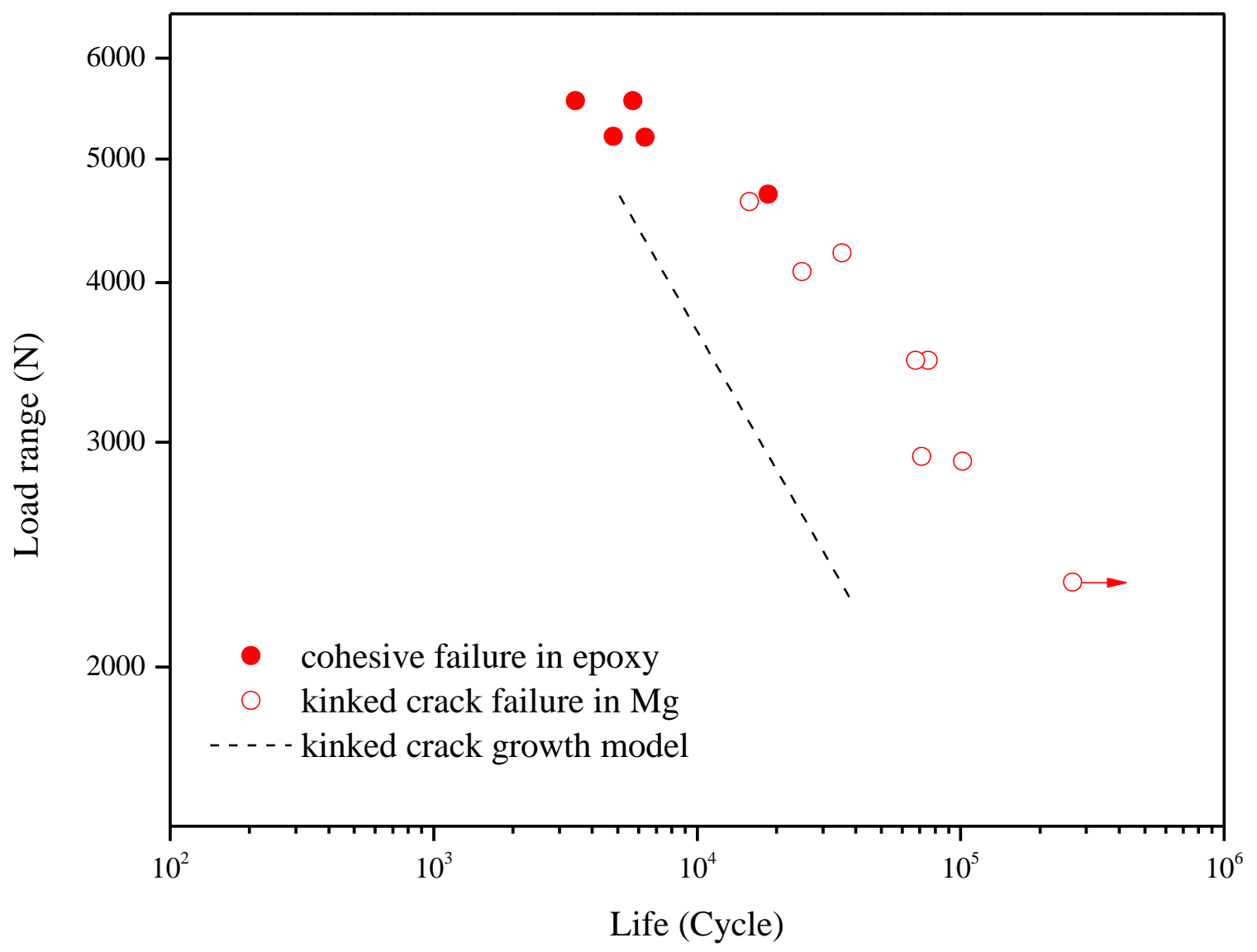

Figure 8. Experimental results of the fatigue tests at the load ratio $\mathrm{R}$ of 0.1 and fatigue life estimations for the adhesive-bonded lap-shear specimens. 